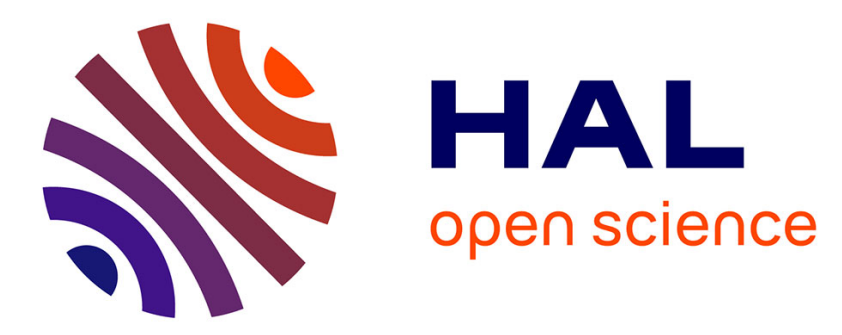

\title{
Determination of the dissolution slowness surface by study of etched shapes I. Morphology of the dissolution slowness surface and theoretical etched shapes
}

\author{
T. Leblois, C. Tellier
}

\section{- To cite this version:}

T. Leblois, C. Tellier. Determination of the dissolution slowness surface by study of etched shapes I. Morphology of the dissolution slowness surface and theoretical etched shapes. Journal de Physique III, 1992, 2 (7), pp.1259-1286. 10.1051/jp3:1992177 . jpa-00248801

\section{HAL Id: jpa-00248801 https://hal.science/jpa-00248801}

Submitted on 1 Jan 1992

HAL is a multi-disciplinary open access archive for the deposit and dissemination of scientific research documents, whether they are published or not. The documents may come from teaching and research institutions in France or abroad, or from public or private research centers.
L'archive ouverte pluridisciplinaire HAL, est destinée au dépôt et à la diffusion de documents scientifiques de niveau recherche, publiés ou non, émanant des établissements d'enseignement et de recherche français ou étrangers, des laboratoires publics ou privés. 
Classification

Physics Abstracts

$81.60-61.50-82.20 \mathrm{P}$

\title{
Determination of the dissolution slowness surface by study of etched shapes
}

\section{Morphology of the dissolution slowness surface and theoretical etched shapes}

\author{
T. Leblois and C. R. Tellier \\ Laboratoire de Chronométrie, Electronique et Piézoélectricité, Ecole Nationale Supérieure de \\ Mécanique et des Microtechniques, La Bouloie, Route de Gray, 25030 Besançon Cedex, France
}

(Received 21 November 1991, accepted 26 March 1992)

\begin{abstract}
Résumé. - Nous avons modélisé le processus de dissolution anisotrope des cristaux en vue d'une application à la simulation des formes obtenues par photolithogravure chimique. La principale originalité de ce modèle tient à l'introduction de tenseurs de dissolution pour exprimer la surface représentative de la lenteur de dissolution. La connaissance de l'équation de la lenteur de dissolution permet de calculer les trajectoires des différents éléments constituant la surface de départ puis de reconstituer par simulation la forme dissoute. Les simulations démontrent que les formes limites des cristaux dissous sont corrélées aux extrema de la lenteur de dissolution. La détermination de la surface de la lenteur se faisant à partir de mesures expérimentales, nous nous sommes efforcés de montrer toutes les difficultés attachées à cette analyse.
\end{abstract}

\begin{abstract}
We propose a theoretical model for the anisotropic etching of crystals, in order to be applied in the micromachining. The originality of the model is due to the introduction of dissolution tensors to express the representative surface of the dissolution slowness. The knowledge of the equation of the slowness surface allows us to determine the trajectories of all the elements which compose the starting surface. It is then possible to construct the final etched shape by numerical simulation. Several examples are given in this paper which show that the final etched shapes are correlated to the extrema of the dissolution slowness. Since the slowness surface must be determined from experiments, emphasis is placed on difficulties encountered when we correlate theory to experiments.
\end{abstract}

\section{Introduction.}

During the last four decades the interest in the etching of crystals to obtain a measure of crystal perfection has been revived [1-9]. Because of its simplicity the etch pit method is commonly used to detect dislocations on semiconductor surfaces $[1-3,8]$ or on quartz surfaces $[4,5,7]$. It has been shown $[1-4,8]$ that the shape of an etch pit or an etch hillock is intimately 
connected with the symmetry of the crystal on which it is formed. It is the reason why it is possible to use etching technique to determine with a good accuracy (half a degree) [9] the orientation of a crystal surface provided the etchant causes the development of sharp and contrasting etch pits. But since a crystal surface is not perfectly smooth the chemical attack results also in the formation of dissolution figures $[1-3,5,7,10-19]$ whose geometrical features change with the crystal orientation. A great number of works $[5,7,10-19]$ have been devoted to quartz crystals and it has been observed that the topology of etched surfaces is very sensitive to the surface orientation. Works [1-3] related to silicon surfaces are generally limited to $\left\{\begin{array}{lll}1 & 0 & 0\end{array}\right\},\left\{\begin{array}{lll}1 & 1 & 0\end{array}\right\}$ and $\left\{\begin{array}{lll}1 & 1 & 1\end{array}\right\}$ surfaces. Finally a small portion of experimental works [20-22] has been primarily concerned with the limiting shapes of starting cylindrical hollows or cylindrical crystals.

In the past few years several attempts [23-28] have been made to apply the photolithographic process to the micromachining of mechanical devices which constitute the sensing element of quartz sensors [24-28] or of silicon integrated sensors [23, 24]. New procedures to analyse the dissolution shapes of crystals have been proposed [25-34]. They include on the one hand, examination of the cross-sectional shapes and evaluation of the lateral underetch resulting from etching processes at a mask edge [25, 29-32] and on the other hand, study of the corner undercutting [33,34]. More recently some workers have investigated more complex structures such as star-like structures [35-37]. Also some methods have been proposed to reduce corner undercutting [38-41].

Over the past few years the prediction of etching shapes has often been based on the kinematic model proposed by Frank [42, 43] which gives us the necessary tools $[43,44]$ to construct geometrically the etching shapes encountered in localized etching. Recently, Tellier and co-workers [45-48] have suggested that it is possible to predict the exact geometrical configuration of etched micromechanical devices using a tensorial analysis of the anisotropic dissolution. This may be done as soon as we can extract with sufficient accuracy the shape of the dissolution slowness surface from etch data. Thus the purpose of this paper is to propose a procedure for deriving the dissolution slowness surface. A numerical simulation is used to show how various theoretical dissolution shapes are correlated to the extrema of the dissolution slowness. A method for generating the dissolution slowness surtace is then presented and emphasis is placed on the necessity of undertaking complementary experiments to overcome some difficulties due to the complexity of the problem.

\section{Kinematic descriptions of the anisotropic dissolution process.}

In the present section the theoretical works dealing with the theories of anisotropic dissolution are surveyed. After summarizing the earlier bi-dimensional models [1-3, 42, 43] proposed to explain the formation of etch pits the attention is confined on recent developments [44-48] which can provide more easy theoretical prediction of dissolution shapes.

In bi-dimensional kinematics theories a surface profile can be represented by a succession of linear profile elements, $\Delta r_{l}$, [42-44] (Fig. 1a). The analysis is generally performed under the assumption that the etching process is only governed by the orientation, $\alpha_{i}$, of surface profile elements, i.e. on the local slope. Then, as the etching time $t$, increases, each profile element moves within the crystal along a straight line called the characteristic trajectory [1, 42, 43]. Some theoretical works have given evidence that the displacement of moving profile element can be evaluated as soon as the polar graph, $V_{\mathrm{N}}(\gamma)$, of the normal etch rate [21] or the « reluctance plot $», 1 / V_{\mathrm{N}}(\gamma),[22,43]$ are known.

In the past few years Tellier and co-workers [44-46] proposed a method directly derived from the Frank's model $[42,43]$ in which two vectors are introduced corresponding to two 

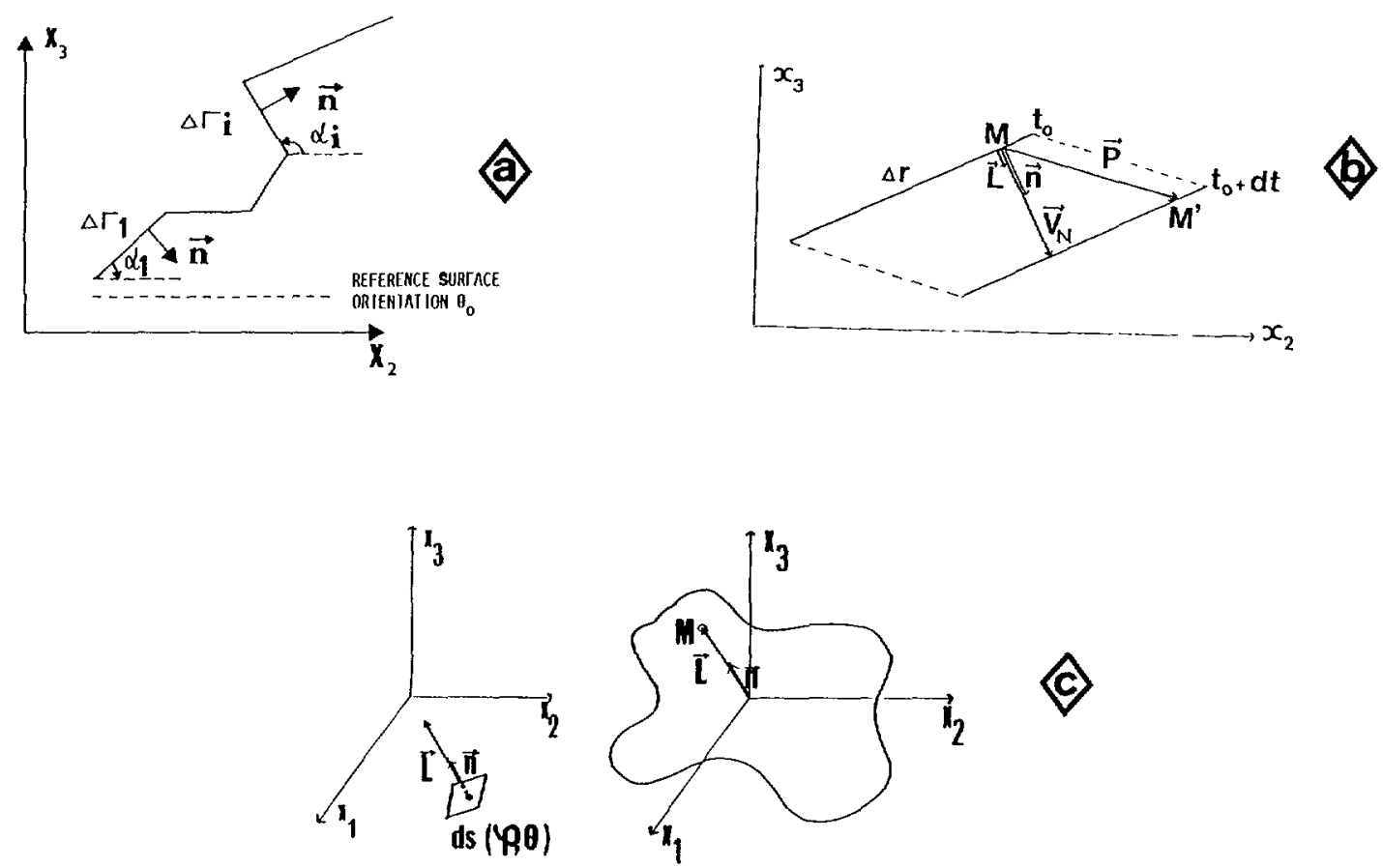

Fig. 1. - Geometry of the model : (a) Representation of a surface profile; (b) Definition of the propagation vector; (c) Three-dimensional representation.

physical measurable quantities namely the propagation vector, $\mathbf{P}$, and the dissolution slowness vector, $\mathbf{L}$. Turning to figure $1 \mathbf{b}$ the propagation vector, $\mathbf{P}$, of a moving profile element, $\Delta r$, is just the vector $\mathbf{M M}^{\prime}$ which, in a kinematic geometric model of the chemical etching is at a fixed etching time, only determined by the orientation of the profile element, $\Delta r$. As suggested earlier by Frank who referred to a « reluctance diagram » to construct the etched shape of a bi-dimensional crystal [43] it is of interest to associate to each profile element a dissolution slowness vector, $L$, whose magnitude, $L(\gamma)$, is the reciprocal of the dissolution rate, $V_{\mathrm{N}}(\gamma)$, and whose positive direction coincides with that of the inward normal unit vector, $\mathbf{n}$, to the profile element [44-45]. Several works [43, 44] give evidence that the propagation vector, $\mathbf{P}$, lies perpendicular to the vector, $\mathbf{T}$, tangent to the polar diagram, $L(\gamma)$, of $\mathbf{L}$ at the joint of corresponding orientation. Using the geometrical properties of vectors $\mathbf{T}$ and $\mathbf{L}$ the cartesian components $\left(\mathrm{d} x_{3}, \mathrm{~d} x_{2}\right)$ of the propagation vector, $\mathbf{P}$ are found to be $[44]$

$$
\begin{aligned}
\mathrm{d} x_{2} & = \pm \frac{B(\gamma)}{(F(\gamma))^{2}} \cdot \mathrm{d} t \\
\mathrm{~d} x_{3} & =-\frac{A(\gamma)}{B(\gamma)} \cdot \mathrm{d} x_{2}
\end{aligned}
$$

where $\gamma$ is the polar angle of $\mathbf{L}$ and $A(\gamma)$ and $B(\gamma)$ denote respectively the cartesian components of $\mathbf{T}$ which here is seen as the derivative of the rotating vector $\mathbf{L}$ with respect to the polar angle $\gamma$. The orientation dependence of $\mathbf{L}$ si given by

$$
\mathbf{L} \cdot \mathbf{L}=[F(\gamma)]^{2}
$$


and the choice between plus or minus sign in equation (1) is determined by the value of the angle that $\mathbf{T}$ makes with the slowness vector $\mathbf{L}$.

These bi-dimensional models can be easily extended to the three-dimensional case as recently shown by Tellier et al. [46]. In this case we suppose that the surface of the crystal can be decomposed into a succession of planar surface elements corresponding to crystallographic faces ( $h k l$ ). Then we try to track the displacement of a moving surface element in space $\left(x_{1}, x_{2}, x_{3}\right)$. Adopting the convention specified in the I.E.E.E. standards on piezoelectricity [49] the orientation of an oriented surface element, ds, is defined by means of two angles of cut $(\varphi, \theta)$ since $\mathrm{ds}$ is determined by two successive rotations :

1) a rotation of $\varphi$ degrees about the $x_{3}$ axis which except for the triclinic crystal system coincides with the $c$ or $a_{3}$ axis of the natural coordinates system provided by the crystal itself ;

2) a rotation of $\theta$ degrees about the $x_{1}$ axis i.e. in most crystal system the $a$ or $a_{1}$ axis.

As the orientation $(\varphi, \theta)$ of the surface element varies the extremity of the dissolution slowness vector generates a representative surface called the dissolution slowness surface [45]. Provided that $L$ depends only on the local orientation $(\varphi, \theta)$ of the surface element it has been found [46] that here again the surface element propagates along a straigth line. Then the trajectory of the surface element can be completely characterized by a propagation vector $\mathbf{P}$ (Fig. 1c) of components $\mathrm{d} x_{1}, \mathrm{~d} x_{2}$ and $\mathrm{d} x_{3}$.

Dealing with a polar representation of $\mathbf{L}$ the cartesian components of the dissolution slowness vector are expressed as

$$
\begin{aligned}
& L_{1}=F(\alpha, \beta) \sin \beta \cos \alpha \\
& L_{2}=F(\alpha, \beta) \sin \beta \sin \alpha \\
& L_{3}=F(\alpha, \beta) \cos \beta
\end{aligned}
$$

with

$$
\mathbf{L} \cdot \mathbf{L}=[F(\boldsymbol{\alpha}, \boldsymbol{\beta})]^{2} .
$$

The two derivatives of $\mathbf{L}$ with respect to the polar angles $\alpha$ and $\beta$ are two vectors $\mathbf{C}_{\alpha}$ and $\mathbf{C}_{\beta}$ which define a plane, $\mathscr{P}$, tangent to the dissolution slowness surface at the point of corresponding orientation $(\alpha, \beta)$. Let the $\left(x_{1}, x_{2}\right)$ plane act as the reference surface and let a planar surface element be a function $x_{3}\left(x_{1}, x_{2}, t\right)$ of three independant variables, the cartesian coordinates $x_{1}$ and $x_{2}$ and the time, $t$, of etching.

It is obvious that the trajectory of a surface element of orientation $(\varphi, \theta)$ (i.e. of orientation $(\alpha, \beta))$ in the $\left(x_{1}, x_{2}, x_{3}\right)$ space follows a straigth line and that the propagation vector $\mathbf{P}$ is now parallel to the normal, $\mathbf{N}$, to plane $\mathfrak{\beta}$ (Fig. 1c). From geometrical considerations the cartesian components of $\mathbf{P}$ are finally found to be [45]

$$
\begin{aligned}
\mathrm{d} x_{1} & =-\left(A_{\beta} \cos \alpha-A_{\alpha} \sin \alpha\right) \mathrm{d} t \\
\mathrm{~d} x_{2} & =-\left(A_{\alpha} \cos \alpha+A_{\beta} \sin \alpha\right) \mathrm{d} t \\
\mathrm{~d} x_{3} & =\frac{C_{\alpha 2} C_{\beta 3}-C_{\alpha 3} C_{\beta 2}}{C_{\alpha 1} C_{\beta 2}-C_{\alpha 2} C_{\beta 1}} \mathrm{~d} x_{1}
\end{aligned}
$$

with

$$
\begin{aligned}
& A_{\alpha}=C_{\alpha 3}\left[(F(\alpha, \beta))^{2} \sin \beta \cos \beta\right]^{-1} \\
& A_{\beta}=C_{\beta 3}[F(\alpha, \beta)]^{-2}
\end{aligned}
$$

where $C_{\alpha_{2}}$ and $C_{\beta i}(i=1,2,3)$ are the cartesian components of the two vectors $\mathbf{C}_{\alpha}$ and $\mathbf{C}_{\beta}$. 
The problem is now to evaluate the various propagation vectors $\mathbf{P}$ associated with all the surface elements composing the starting crystal shape, to distinguish between diverging and converging trajectories $[43,44]$ and then to eliminate parts of the flat or curved faces which disappear with prolonged etching. Such an evaluation requires the knowledge of the equation which gives the orientation dependence, $L(\varphi, \theta)$, of the magnitude of the dissolution slowness vector. To formulate $L(\varphi, \theta)$ we can start with a tensorial representation of the dissolution slowness recently proposed by Tellier et al. [45].

Taking into account that the cartesian components of the inward normal unit vector, $n$, are given by

$$
\begin{aligned}
& n_{1}=\sin \varphi \cos \theta \\
& n_{2}=-\cos \varphi \cos \theta \\
& n_{3}=-\sin \theta
\end{aligned}
$$

the representative equation of the slowness surface can be expressed in terms of a polynomial relation involving the three components of $\mathbf{n}$.

As a result the magnitude $L(\varphi, \theta)$ is related to $n_{1}, n_{2}$ and $n_{3}$ by means of the components $D_{0}, D_{i}, D_{i j}, D_{i j k}, D_{i j k \ell}$, of dissolution tensors $[45,47]$ :

$$
L(\varphi, \theta)=D_{0}+D_{i} n_{i}+D_{i j} n_{i} n_{j}+D_{i j k} n_{i} n_{j} n_{k}+D_{i j k \ell} n_{i} n_{j} n_{k} n_{\ell}+\cdot
$$

The number of constants necessary to specify the orientation properties of the dissolution slowness surface referred to a given crystal class is reduced by the symmetry the class possesses. This procedure gives a generalized relation for the dissolution slowness $L(\varphi, \theta)$ which reflects the symmetry of the crystal. But it may be observed that modifying the maximum rank of tensors retained in the foregoing procedure can induce changes in the final expression reached for $L(\varphi, \theta)$.

For illustrative purposes two examples of this procedure are discussed. Turning to the symmetry point group 23 for which the symmetry operations can be simply treated by permuting cyclically the subscripts and carrying out the calculations up to tensors of rank 10 we obtain the general relation [47].

$$
L(\varphi, \theta)=S_{1}+S_{2}+S_{3}+S_{1}+S_{2}+S_{3}
$$

with

$$
\begin{aligned}
& S_{1}=\sum_{k=0} a_{k}(\cos \theta \sin \theta)^{2 k} \\
& S_{2}=\sum_{\ell=1} \sum_{m=0} b_{\ell, m}\left(\sin \varphi \cos \varphi \cos ^{2} \theta\right)^{2 \ell}(\cos \theta)^{2 m} \\
& S_{3}=\sum_{L=0} \sum_{M=0} \mathcal{C}_{L, M}(\cos \varphi \cos \theta)^{2 L}(\cos \theta)^{2 M} \\
& S_{1}=\sum_{r=0} c_{r}(\sin 2 \varphi \sin 2 \theta \cos \theta)^{2 r+1} \\
& S_{2}=\sum_{s=0} \sum_{t=0} d_{s, t} \sin \theta\left(\sin \varphi \cos \varphi \cos ^{2} \theta\right)^{2 s+1}(\cos \theta)^{2 t} \\
& S_{3}=\sum_{P=0} \sum_{Q=0} \sum_{R=0} \mathfrak{D}_{P, Q, R}\left(\sin \varphi \cos \varphi \sin \theta \cos ^{2} \theta\right)^{2 P+1}(\cos \varphi \cos \theta)^{2 Q}(\cos \theta)^{2 R}
\end{aligned}
$$

The fact that the class 23 is a non-centrosymmetric class is seen through the series $\boldsymbol{s}_{1}, \boldsymbol{S}_{2}$ and $\boldsymbol{S}_{3}$ which involve terms of odd powers. 
It is also of interest to note that the coefficients of the series are expressed in terms of the independent dissolution constants [47]. As a consequence they contain the symmetry of the point group and a complete description of the anisotropic properties still demands the specification of relations between these coefficients.

The anisotropy developed in the symmetry point group 32 to which the $\alpha$ quartz crystal belongs is described by means of the general expression:

$$
L(\varphi, \theta)=S_{1}^{*}+S_{1}^{*}+S_{2}^{*}
$$

with

$$
\begin{aligned}
& S_{1}^{*}=\sum_{j=0} A_{j}(\cos \theta)^{2 j} \\
& S_{1}^{*}=\sum_{k=1} B_{k}(\cos \theta)^{2 k+1} \sin 3 \varphi \\
& S_{2}^{*}=\sum_{\ell=1} C_{\ell}(\cos \theta)^{2 \ell+1} \sin \theta \cos 3 \varphi
\end{aligned}
$$

which is obtained by neglecting the dissolution constants which appear in tensors of rank higher than 6. Thus we cannot ignore that following the procedure up to tensors of rank 10 will certainly give complementary terms. Moreover here again the coefficients $A_{J}$, $B_{k}$ and $C_{\ell}$ must satisfy complementary conditions imposed by the crystal symmetry.

Examining equations (17) to (20) it can be seen that these equations verify some symmetry operations of the point group 32 in which $x_{3}$ is a symmetry triad axis 3 and $x_{1}$ a binary axis 2 . Effectively taking $\varphi=0$ the general equation contains as expected only even terms in $\sin \theta$ and/or $\cos \theta$. Moreover it is obvious that terms in the form $\sin 3 \varphi$ or $\cos 3 \varphi$ reflect the operation 3 along the direction $x_{3}$ which leaves the generalized equation unchanged.

Difficulties arise because the generalized equations involve unknown coefficients connected to dissolution constants which need to be determined experimentally if we wish to find a satisfactory representation of the dissolution slowness surface. The connection between experimental works and the «true» dissolution constants remains a task of considerable difficulty because these constants cannot be observed directly. In practice the constants can be quantitatively deduced only by combining different possibilities of measuring the dissolution slowness for some particular values of the angles of cut. Effectively in most cases these measurements are only reliable if they are associated to extrema in the dissolution slowness, $L(\varphi, \theta)$. Such a condition restricts considerably the number of interesting quantitative data. One may argue that the simpler method to evaluate $L(\varphi, \theta)$ is to measure the normal dissolution rate $V_{\mathrm{N}}(\varphi, \theta)$. For this purpose it is usual $[5,10,11,14,15]$ to start with differently oriented parallel plate specimens and to measure the crystal thickness after repeated isothermal etchings. But it should be remarked that this method presents several disadvantages :

(i) the determination of the law $V_{\mathrm{N}}(\varphi, \theta)$ requires a great number of specimens ;

(ii) it concerns only the orientations $(\varphi, \theta)$ for which the etch rates are the same for the two faces ;

(iii) the initial surface damage can affect the etch rate in the first stages of etching $[12,50]$. Then the specimens must suffer a prolonged etching and the two faces are often covered by dissolution figures of relatively large dimensions $[10,11,50]$;

(iv) Frozen-in defects can also markedly influence the dissolution process. As a consequence ideally the specimen must be cut in the same zone of the crystal blank.

Then to provide a satisfactory description of the anisotropic dissolution process we need to undertake various complementary experiments including for example studies of surface 
profilometry traces $[44,45]$, of surface topography by scanning electron microscopy, of the etched shape of grooves $[1,29-32,48]$ and of the out-of-roundness profiles $[20-22,48]$. In this paper an attempt is made to give more informations on these possibilities to obtain reliable data. For this purpose we examine theoretically how some etched profiles are correlated to the topology of the dissolution slowness surface. Finally we suggest some more elegant use of data to derive a quite correct slowness surface together with restricting the number of differently oriented plates necessary for this determination.

\section{Theoretical prediction of etched shapes.}

3.1 Dissolution CRITERIA. - Batterman [51] is the first to propose two criteria to set conditions on the formation of etch pits and etch hillocks. These conditions can be summarized as follows : an etch pit will form when the local etching is slower than that of the reference surface and conversely a local etching faster than that of the reference surface will cause the development of a hillock. Further Irving [2] applied these two criteria to state conditions for the stability during the dissolution of the intersection between two surface profile elements and to deduce surface profile features from etch rate $V_{\mathrm{N}}, v s$. orientation, $\alpha$, plots. Here to avoid some confusion in the discussion of results it is more appropriate to express the arguments developed by Irving in terms of the dissolution slowness, $L(\varphi, \theta)$. Now we consider two intersecting planes $\mathfrak{T}_{1}$ and $\mathfrak{T}_{2}$ limiting locally the starting crystal surface : a concave intersection is stable provided there is no plane between them with a higher dissolution slowness and conversely a convex intersection remains stable when there is no plane between them with a lower dissolution slowness.

For the two converse situations etching causes the formation of rounded surfaces composed of an assemblage of all planes lying within $\mathfrak{T}_{1}$ and $\mathfrak{S}_{2}$. For example it is just the result one may observe when a maximum of $L(\varphi, \theta)$ occurs for an orientation $\left(\varphi_{\mathrm{m}}, \theta_{\mathrm{m}}\right)$ corresponding to a plane $\mathfrak{T}_{\mathrm{m}}$ lying between the two planes $\mathfrak{T}_{1}$ and $\mathfrak{T}_{2}$ forming the starting concave intersection.

Care must be taken that stable intersections imply converging trajectories of the two initial surface elements. Diverging trajectories produce the formation of rounded or curved intersections in the first stage of etching. The extent of limiting faces forming the rounded surface increases with more and more diverging trajectories. If a concave (convex) intersection includes several accentuated maxima (minima) of $L(\varphi, \theta)$ prolonged etching results in the development of limiting planar faces whose orientations $\left(\varphi_{\mathrm{e}}, \theta_{\mathrm{e}}\right)$ are just those associated with these extrema.

Various initial surface profiles can be treated in the framework of dissolution criteria to yield rapidly approximate dissolution shapes $[20-22,29,30,35,48]$. From the preceding we can effectively infer that

(i) the final cross-sectional etched shape of an initial cylindrical hollow (i.e. of an assemblage of concave intersections) is limited by the planes associated with the maxima of $L(\varphi, \theta)$. The same situation occurs when we are concerned with the profiles produced by localized etching of a crystal at a mask edge $[29,30,48]$;

(ii) the final etched cross-section of a starting cylindrical crystal is essentially determined by the presence of minima in $L(\varphi, \theta)$. Since mesa also present convex corners when etched for example with a square mask pattern corner undercutting with beveling faceting planes can also take place. Something of the sort is actually observed in experiments [38-41] on (100) oriented silicon wafers where beveling planes are frequently identified as $\{212\}$ planes connected with minima in $L(\varphi, \theta)$.

Conclusions (i) and (ii) may serve to construct geometrically dissolution profiles which are assumed to be formed by limiting planar facets associated with one type of extrema. This 
approximate procedure ignores the role played by extrema of converse nature in the development of rounded profiles. Then the accuracy of the geometrical method was recently discussed [48]. Specially the influence of he relative amplitude of successive minima and maxima of $L(\varphi, \theta)$ on the extent of successive limiting facets was partially studied. The main conclusions relative to the shape of etched profiles are drawn starting from polar diagrams of the dissolution slowness with defined features. But here the problem is : how can we use experimental dissolution profiles to deduce with a good approximation the topology of the dissolution slowness surface? To answer this question in the part I of the present paper we analyse theoretical etched shapes of cross-sectional and surface profiles in order to appreciate the features which remain essential to arrive at a quite correct prediction of the slowness surface topology. Complementary treatments of experimental data by which it may be improved are also outlined.

\subsection{DisSOLUTION SHAPES.}

3.2.1 Cross-sectional profiles. - Since we can reconstruct the slowness surface by determining successive polar diagrams of the form $L\left(\varphi, \theta=0^{\circ}\right)$ or $L\left(\varphi=0^{\circ}, \theta\right)$ it seems appropriate to investigate theoretically the changes in shape with repeated etchings of initially circular hollows or crystals.

For this purpose let us consider the case of an hypothetical crystal belonging to the symmetry point group 23 for which the representative surface of the dissolution is given by equation (10).

For convenience we have chosen on the one hand for the unknown cefficients, values which leave the slowness surface crudely inscribed in a sphere and on the other hand for the higher rank of tensors, $N_{\max }$, a value of 10 which ensures a sufficiently complicated topology [47]. Figure 2 shows polar diagrams of $\mathbf{L}$ contained in a doubly rotated plane whose orientation is defined by means of two angles $\varphi, \psi$. In this case the dissolution slowness vector $L$ lies in the plane whose normal $\mathbf{N}$ coïncides with the transformed axis $x_{2}^{\prime \prime}$ which is obtained by two successive rotations (Fig. $2 \mathrm{~d}$ ) :

(i) a rotation of $\varphi$ degrees about the $x_{3}$ axis which changes $x_{1}$ and $x_{2}$ axes in $x_{1}^{\prime}$ and $x_{2}^{\prime}$ axes ;

(ii) a rotation of $\psi$ degrees about the $x_{2}^{\prime}$ axis.

Then it is left as an exercice for the reader to verify that in the case where $\varphi=45^{\circ}$ and $\psi=144^{\circ}$ the $x_{2}^{\prime}$ axis is just normal to a $\{111\}$ plane. Hence in this case we are concerned with a threefold rotation axis and the polar diagram must satisfy the symmetry operation 3. From a rapid examination of figure $3 \mathrm{~b}$ we observe a complete fulfillment of this condition.

The etched shapes of bi-dimensional crystals were numerically determined. The program has been described elsewhere [48]. However two points merit to be outlined.

1) The first step consists to determine the orientation $(\varphi, \theta)$ of all the potentially present elements of the starting profile.

2) Tests are used in the last step to distinguish between diverging and converging trajectories and finally to eliminate the etched elements which make no contribution to the final dissolution profile.

The step 1 remains essential for the polar diagrams displayed in figure 2 for which it is clear that any element incorporated in the starting circular section corresponds to a doubly-rotated plane.

The theoretical dissolution shapes of starting cylindrical hollow and crystal are respectively given in figures 3 and 4 . First of all we rapidly see that for the orientation $\left(\varphi=45^{\circ}\right.$, 

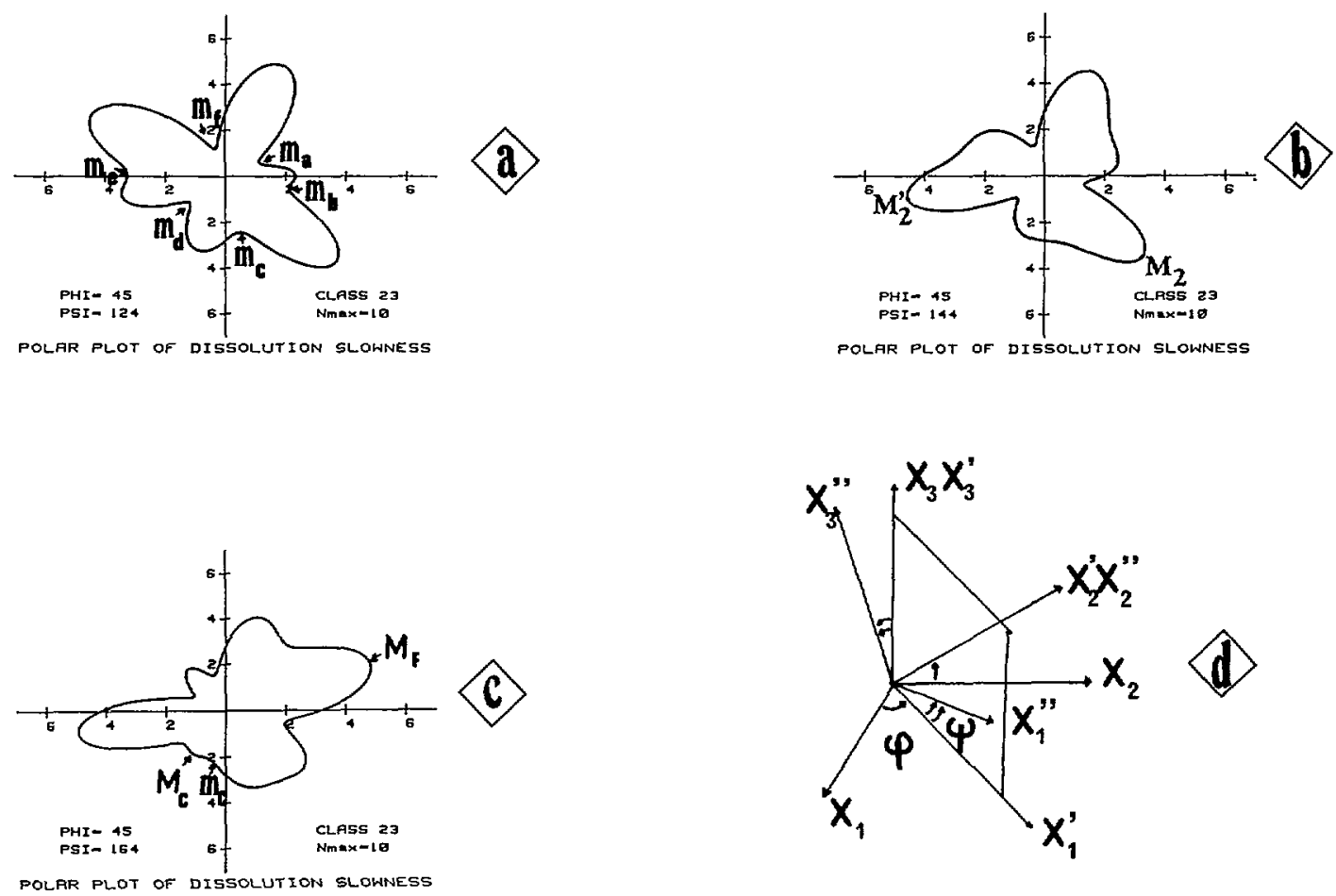

Fig. 2. - Polar diagrams of the dissolution slowness relative to a crystal belonging to point group 23. (a) $\varphi=45^{\circ}, \psi=124^{\circ}$; (b) $\varphi=45^{\circ}, \psi=144^{\circ}$; (c) $\varphi=45^{\circ}, \psi=164^{\circ}$; (d) Definition of the angles $\varphi$ and $\psi$.

$\psi=144^{\circ}$ ) the etched sections present as expected a three-fold symmetry. Secondly a complete examination of figures 3 and 4 reveals the following features.

(i) Starting with a cylindrical hollow (Fig. 3) somewhat planar facets connected with maxima of $\mathbf{L}$ develop progressively with repeated etching. Moreover the facets that can be qualified of planar limiting facets are generally associated with secondary maxima i.e. they correspond to the fasted formed facets. But one cannot ignore that the limiting facets appears when trajectories of successive elements diverge and that consequently an earlier development of facets is also correlated to a rapid divergence of trajectories.

(ii) Turning to cylindrical crystal it can be seen (Fig. 4) that all the planar or rounded faceted regions produced by etching correspond to minima in $L$. At a fixed etching time the quasi-planar limiting facets are correlated to minima minimora in $\mathbf{L}$ whereas secondary minima cause the formation of rather rounded facets (see for example regions $e$ and $c$ in Fig. 4a related to respective minima $m_{\mathrm{c}}$ and $m_{\mathrm{c}}$ in Fig. 2a). The minimum $m_{\mathrm{c}}$ in figure $2 \mathrm{c}$ appears as a small perturbation but it yields also a distinct rounded facet $c$ (Fig. 4c).

From these observations we can conclude that from a general point of view and independently of the nature of the initial section :

(a) When starting with a cylindrical crystal the extent of facets increases with decreasing magnitude, $L$, of the dissolution slowness vector. It is sufficient for example to compare facets $\mathrm{e}$ and $\mathrm{f}$ in figure $4 \mathrm{a}$ with facet $\mathrm{a}$. The converse situation results from etching of cylindrical hollows (look, for example, at facets $\mathrm{c}$ and $\mathrm{f}$ in Fig. $3 \mathrm{c}$ respectively associated with maxima $M_{\mathrm{C}}$ and $M_{\mathrm{F}}$ in Fig. $2 \mathrm{c}$ ). Moreover the flatness of a facet is improved with decreasing $L$. 


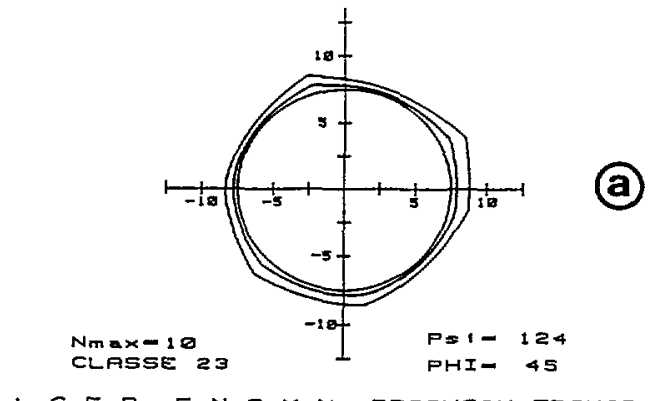

L.C.E.P. E.N.S.M.M. BESANCON.FRANCE

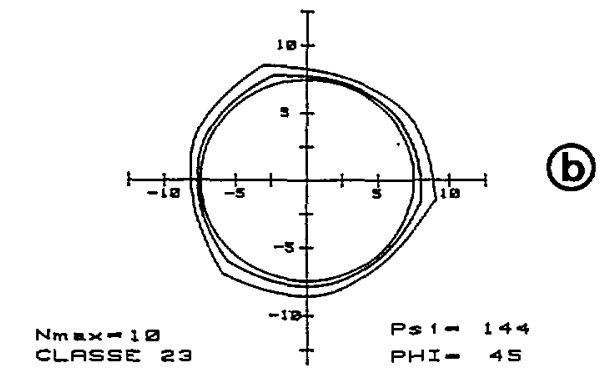

L.C.E.P. E.N.S.M.M. BESANCON,FRANICE

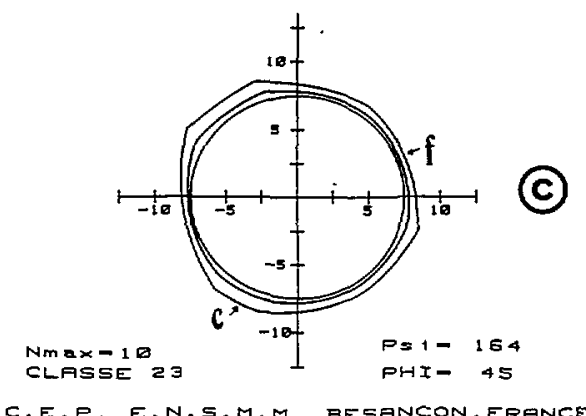

Fig. 3.

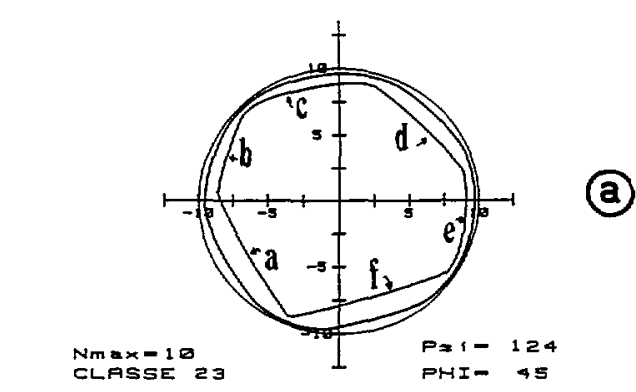

L.C.E.P. E.N.S.M.M. EESANCON.FRANCE

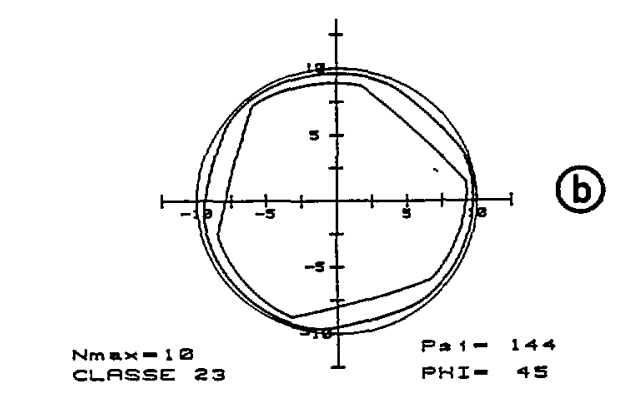

L.C.E.R.,E.N.S.M.M. BESANCON, FRANCE

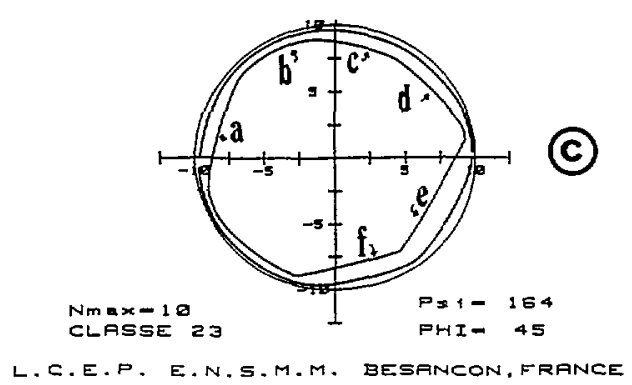

Fig. 4.

Fig. 3. - Etched shapes of starting cylindrical hollows for two etching times $t=1, t=3$ a.u. (a) $\varphi=45^{\circ}, \psi=124^{\circ}$; (b) $\varphi=45^{\circ}, \psi=144^{\circ}$; (c) $\varphi=45^{\circ}, \psi=164^{\circ}$.

Fig. 4. - Etched shapes of starting cylindrical crystals for two etching times $t=1, t=3$ a.u. (a) $\varphi=45^{\circ}, \psi=124^{\circ}$; (b) $\varphi=45^{\circ}, \psi=144^{\circ} ;$ (c) $\varphi=45^{\circ}, \psi=164^{\circ}$.

(b) The shape of dissolution profiles changes with the duration of etching. With repeated etchings the planar facets grow at the expense of the curved regions. Let be $I_{p c}$ the intersection between two successive curved and flat regions and $\alpha$, the angle that $\mathrm{OI}_{\mathrm{pc}}$ makes with the horizontal axis. It is obvious that $\alpha_{i}$ varies with the etching time. This variation illustrates the instability of the successive intersections of the curved region which are formed by elements with diverging trajectories. The etched profiles displayed in figures 3 and 4 are thus in total agreement with the dissolution criteria. 
The study of the changes in roundness of initially circular sections on etching constitutes a complementary way for obtaining informations useful for the derivation of the dissolution slowness surface. In this case the program generates the least square circle and then calculates and draws magnified out-of-roundness profiles.

The out-of-roundness profiles shown in figures 5 and 6 present successive hills and valleys. In figure 5 valleys are generated by portions of polar graphs in the vicinity of minima of $\mathbf{L}$ whereas in figure 6 they are associated with maxima. The bottoms $B$, of valleys are just produced by extrema of $\mathbf{L}$ connected with limiting facets. With repeated etchings these bottoms move along straight line segments which pass through the center $O$ of the least-
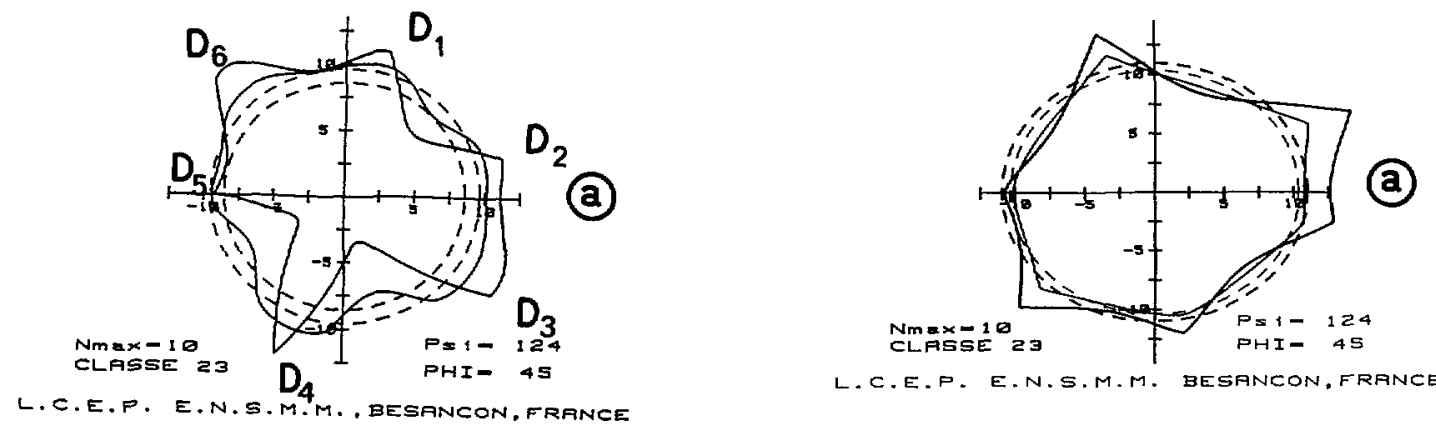

L.C.E.P. E.N.S.M.M. BESANCON,FRANCE

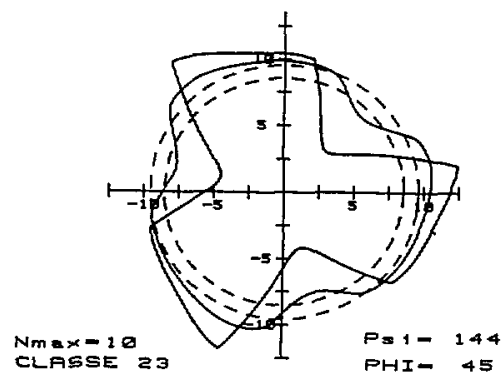

(b)

L.C.E.P. E.N.S.M.M. BESANCON, FRANCE

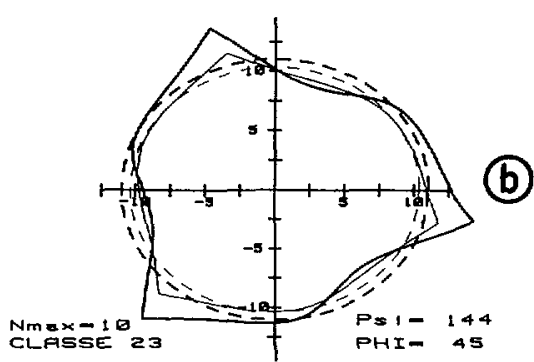

L.C.E.P..E.N.S.M.M. BESANCON,FRPNCE

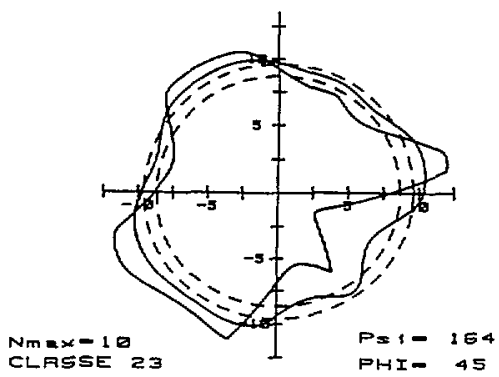

(C)

L.C.E.F. E.N.S.M.M., BESANCON, FRANCE

Fig. 5.

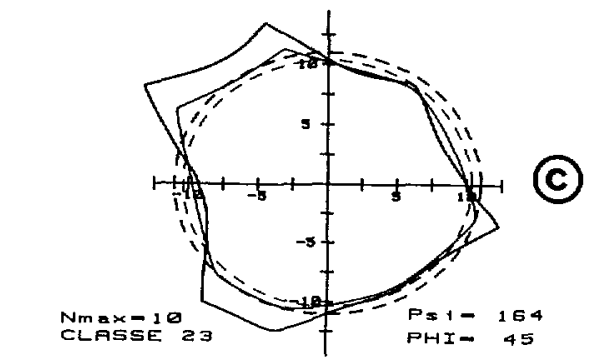

L.C.E.P. E.N.S.M.M.. BESANCON,FRANCE

Fig. 6.

Fig. 5. - The out-of-roundness profiles associated with the etched shapes shown in figure 4.

Fig. 6. - The out-of-roundness profiles associated with the etched shapes shown in figure 3 . 
square circle. This observation significates that as expected the angle $\delta_{i}$ (Fig. 5b) which characterizes the orientation of the surface element associated with a fixed bottom remains invariant. Moreover comparison of figure 2 with figure 5 shows off that inversing the profiles ( $180^{\circ}$ rotation) and ignoring the relative amplitude of successive maxima and minima in polar diagrams the out-of-roundness profiles constitute a crude approximation of polar graphs. It should be mentionned that the out-of-roundness profiles correspond to the inner surface of the crystal in figure 5 and to the outer surface in figure 6.

Thus a more realisatic representation of the polar diagram can be achieved in the second case by changing the sign of the deviation with respect to the least square circle of center $O$. Now it is readily apparent (Fig. 7) that the opposite out-of-roundness profiles are directly correlated to the polar diagram even if the relative dissolution slowness extrema are not yet respected.

It is evident that the two complementary out-of-roundness profiles give us the exact orientation of surfaces for which maxima of $L$ (concave crystals) or minima of $L$ (convex crystals) occurs. It may be of interest to look now at the extrema of oppositive nature which
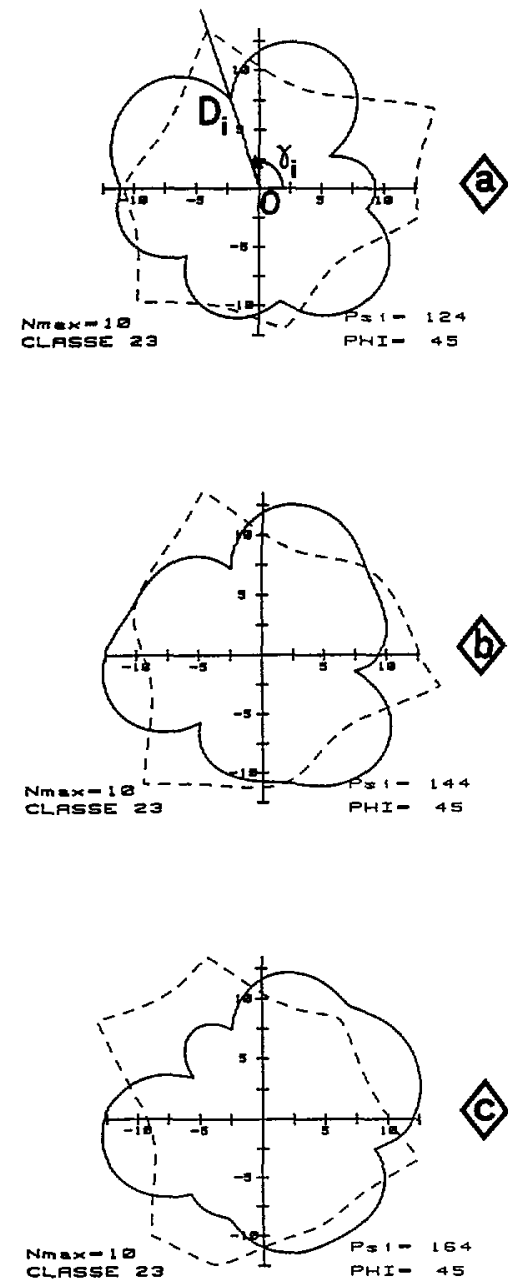

Fig. 7. - Inversion of the out-of-roundness profiles shown in figure 6 . The etching time, $t$, is taken equal to 3 a.u. 
give rise to sharp discontinuities, denoted $D_{i}$, in the out-of-roundness profiles associated with hills in figure 5 and with re-entrant edges in figure 7 . We observe that the angle $Y_{\imath}$ between the line joining $\mathrm{O}$ and $D_{i}$ and the horizontal axis changes in general with incremental etching. These changes are particularly marked for the discontinuities $D_{1}, D_{2}$ and $D_{3}$ appearing in the profiles of figure 8. In contrast the orientation of discontinuities $D_{4}$ and $D_{6}$ seems to remain unchanged with repeated etchings. At this point it becomes useful to specify the condition for obtaining discontinuities whose orientations do not depend of the extent of etching. Comparing carefully figures $2 \mathrm{a}$ and 8 . It appears that the angles $Y_{i}$ keep constant values until we are concerned with portions of the polar graph which exibit quite symmetrical topologies. This geometrical condition is met by valleys formed around the discontinuities $D_{4}$ and $D_{6}$ which are respectively associated with minima $m_{\mathrm{d}}$ and $m_{\mathrm{f}}$ in figure $2 \mathrm{a}$. In contrast asymmetrical portions of polar graphs in the neighbourhood of minima $m_{\mathrm{a}}$ and $m_{\mathrm{b}}$ cause the formation of unstable discontinuities $D_{1}$ and $D_{2}$. But it should be noticed that in any case we put a false interpretation on data if we make tentative to evaluate minima in $L$ from the deviations related to edge discontinuities.

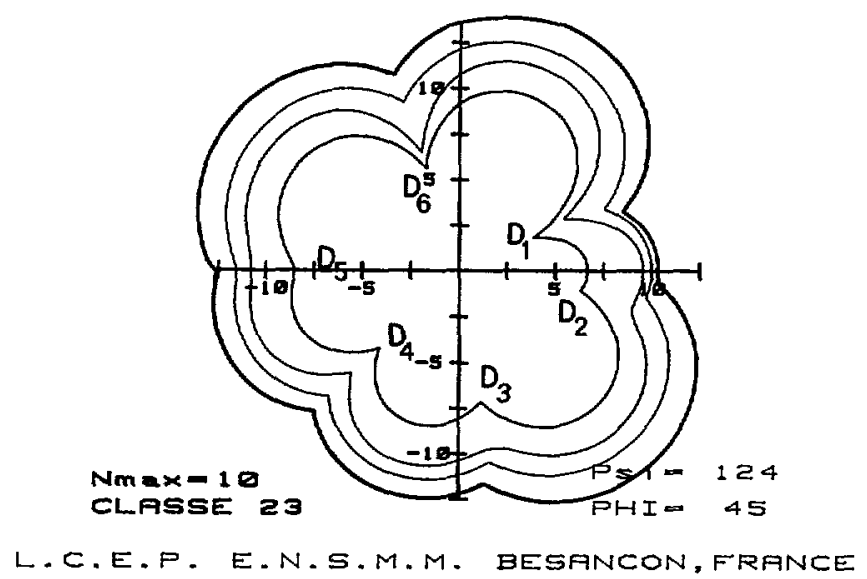

Fig. 8. - Changes in the out-of-roundness profiles with the etching time. The case where $\varphi=45^{\circ}$, $\psi=124^{\circ}$; (a) $t=1$ amplification : $\times 10$; (b) $t=3$ amplification : $\times 5$; (c) $t=5$ amplification : $\times 2.5$; (d) $t=7$ amplification : $\times 1.75$.

3.2.2 Surface profiles. - Following the changes in shape with repeated etchings of starting circular sections as well as looking at the cross-sectional profiles which result of localized etching at a mask edge [29-32] give us some possibilities of determining the orientations for which the slowness surface exhibits extrema and of evaluating the corresponding magnitude of $L(\varphi, \theta)$. We can then by successive iterations represent the slowness surface graphically. This derivation remains a difficult task and requires always several experimental verifications. These experimental checks work with etching of starting circular section [20-22] or with etchings at a mask edge [29-32] rather than with direct measurement of the etch rate of thin differently-oriented plates. However it is also possible to track the evolution of the shape of surface profiles with prolonged etching. Effectively a great number of experimental works have given evidence of a strong dependence of profile shapes on crystal orientation $[10-19,44$, 45]. In this section some attempts are thus made to investigate what useful informations can be extrated from the study of surface textures. For simplicity we start with an initially 
triangular profile to finish with a « real » surface profile as measured with a microprocessorbased profilometer.

3.2.2.1 Triangular surface profiles. - Since we suppose that a surface profile is composed of successive linear elements it may be convenient to consider the case of a triangular profile with angles $\pm \alpha_{\max }$ which exhibits intersections successively convex and concave. To derive the etched profiles we have to consider all the profile elements whose angle $\alpha$ is in the range between $-\alpha_{\max }$ and $+\alpha_{\max }$. Let us look at the particular case where the polar graph of $\mathbf{L}$ presents for a fixed angle close to zero degree a maximum of $L(\varphi, \theta)$ (Fig. 9a). Applying the criteria (i) and (ii) in section 3.1 it is obvious that the trajectories of two profile elements forming a convex intersection converge making the intersection stable whereas diverging trajectories at a concave intersection transform the sharp edge in a curved region. Then in most cases a surface profile which involves intersections of different nature is rarely formed by limiting facets and its shape changes continuously with prolonged etching. In particular the slopes of the various elements composing the etched profile tend to decrease in amplitude with increasing etching. It is the reason why we follow the theoretical changes with incremental etching in the shape of profiles but also in the distribution of slopes.
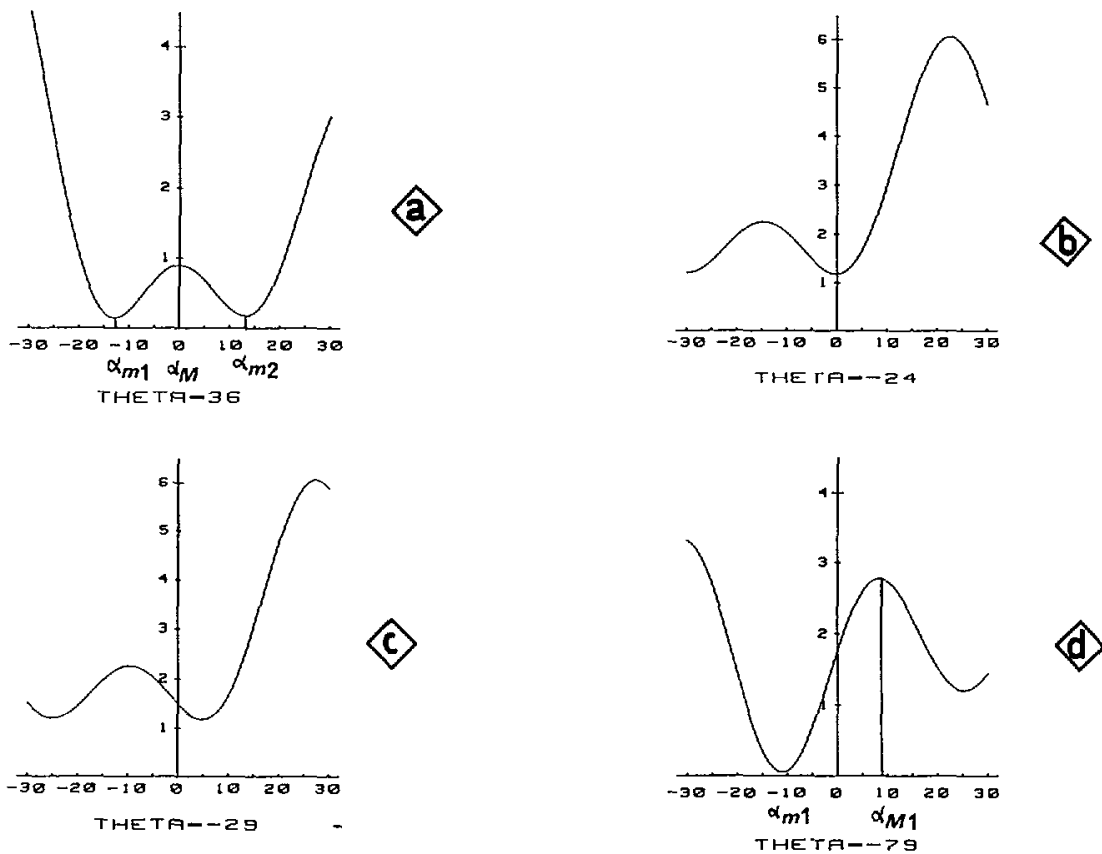

Fig. 9. - Plots of $L$ vs. $\alpha$ : the case of a singly-rotated crystal belonging to the point group 32 . (a) $\theta=36^{\circ}$, (b) $\theta=-24^{\circ}$, (c) $\theta=-29^{\circ}$, (d) $\theta=-79^{\circ}$.

Several theoretical results correlated with the $L(\alpha) v s$. plots shown in figure 9 are displayed in figure 10. Figures 11 to 12 give the corresponding distributions of slopes. Turning to the simpled case (Fig. 9a) where a maximum of $\mathbf{L}$ occurs for an orientation which coincides approximately with that of the reference surface we observe (Fig. 10a) that a curved concave background develops as expected for profiles with $\alpha_{\max }<12^{\circ}$. The profile elements potentially present at an initial concave intersection contribute weakly to the curved portion in the first stage of etching. But with increasing etching time elements with small slopes grow 

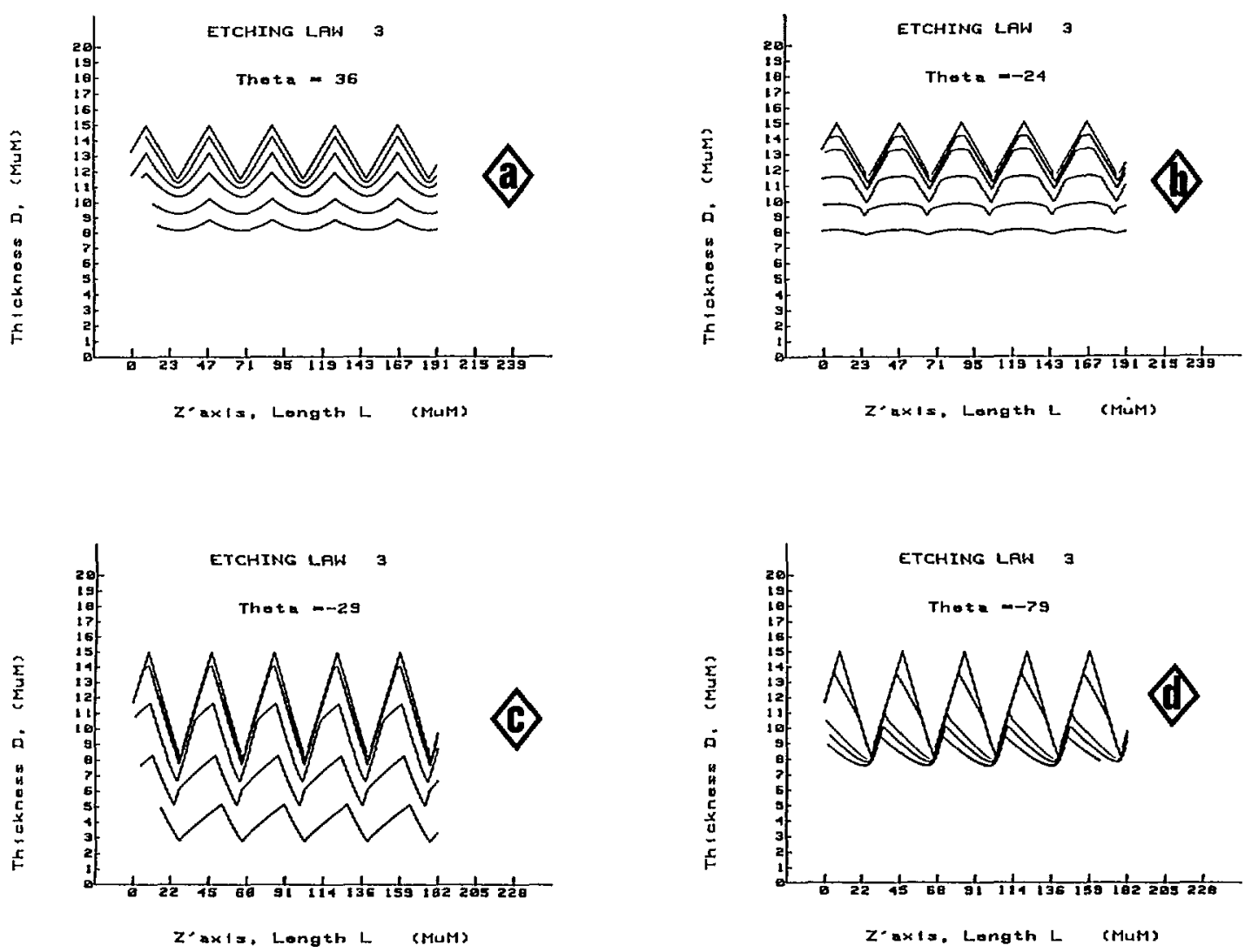

Fig. 10. - Theoretical shapes of etched surface profiles. Thickness and length are given in $\mu \mathrm{m}$ (MuM) : (a) $\theta=36^{\circ}, \alpha_{\max }=10^{\circ}, t=0.2,0.5,1,2,3$; (b) $\theta=-24^{\circ}, \alpha_{\max }=10^{\circ}, t=1,2,4,6,8$; (c) $\theta=-29^{\circ}$, $\alpha_{\max }=20^{\circ}, t=1,4,8,18$; (d) $\theta=-79^{\circ}, \alpha_{\max }=20^{\circ}, t=0.1,0.3,0.6,1$.

at the expense of elements with higher slopes (Fig. 11a). The converse situation (Fig. 9b) leads to a convex background (Fig. 10b). Note that in this case the reference surface etches faster than in the preceding case so that the rounded intersections are found to be composed of element with small slopes (Fig. $1 \mathrm{lb}$ ).

We can also look at the angle $\beta *$ that the trajectory of a profile element makes with the inward normal to the reference surface. This angle defines the direction of the propagation vector $\mathbf{P}$ i.e. characterizes the trajectory of the moving element. When in a fixed range of values for $\alpha$ the angle $\beta *$ passes rapidly for $\alpha=\alpha_{\mathrm{D}}$ from a negative value to a positive value with increasing values of $\alpha$ we can infer that the trajectories of corresponding elements forming a concave intersection diverge. Conversely a variation from a positive value to a negative value on a narrow range of $\alpha$ values characterizes the divergence of trajectories associated with a convex intersection. Figures $13 \mathrm{a}$ and $13 \mathrm{~b}$ illustrate for $\alpha_{\max }=10^{\circ}$ the two converse situations which occurs for $\alpha_{D}$ in the vicinity of $0^{\circ}$.

The $L v s . \alpha$ plot drawn in figure $9 \mathrm{c}$ shows a more complex situation since for $\alpha_{\max }=20^{\circ}$ we are concerned with two successive extrema of different nature : a maximum of $L$ for $\alpha \approx-9^{\circ}$ and a minimum for $\alpha \approx 5^{\circ}$.

Trying to predict the etched shapes in terms of diverging trajectories (Fig. 13c) we can at the first sight conclude that etching results in the formation of profiles with alternate shape 


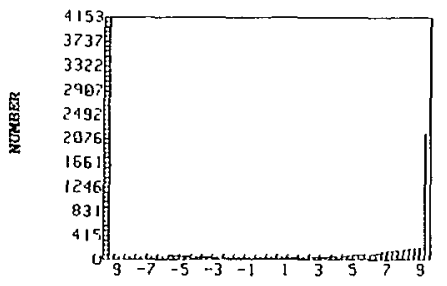

SLOPES (DEGRER)

$\left[\mathbf{a}_{1}\right]$

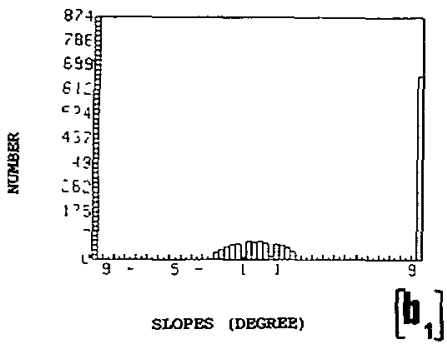

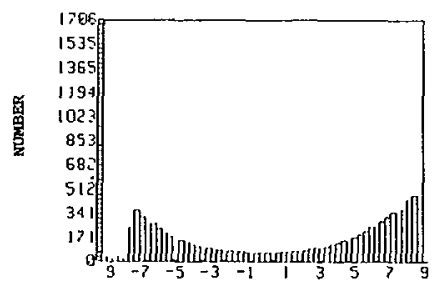

SLOPES (DEGREE)

$\left[\mathrm{a}_{2}\right]$

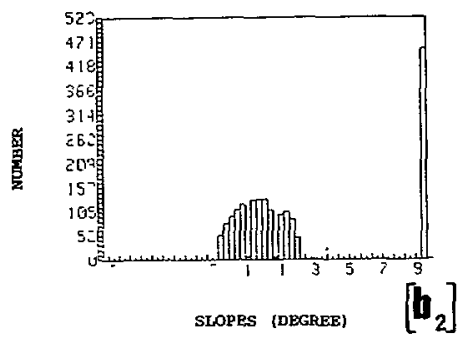

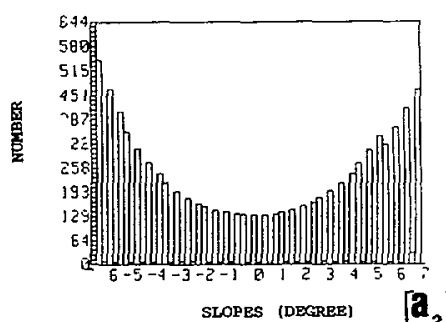

$\left[a_{3}\right]$

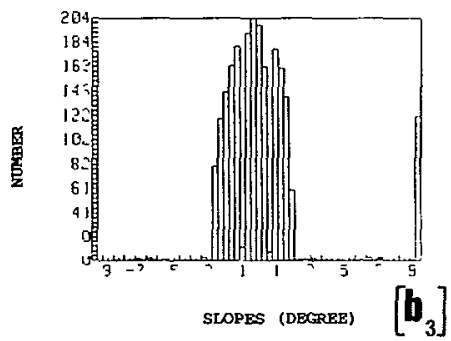

Fig. 11. - Changes in the distribution of slopes with the duration of etching. (a) $\theta=36^{\circ} ;\left(a_{1}\right) t=0.2$, (a) $t=0.5,\left(a_{3}\right) t=1 ;(b) \theta=-24^{\circ} ;\left(b_{1}\right) t=2,\left(b_{2}\right) t=4,\left(b_{3}\right) t=6$.
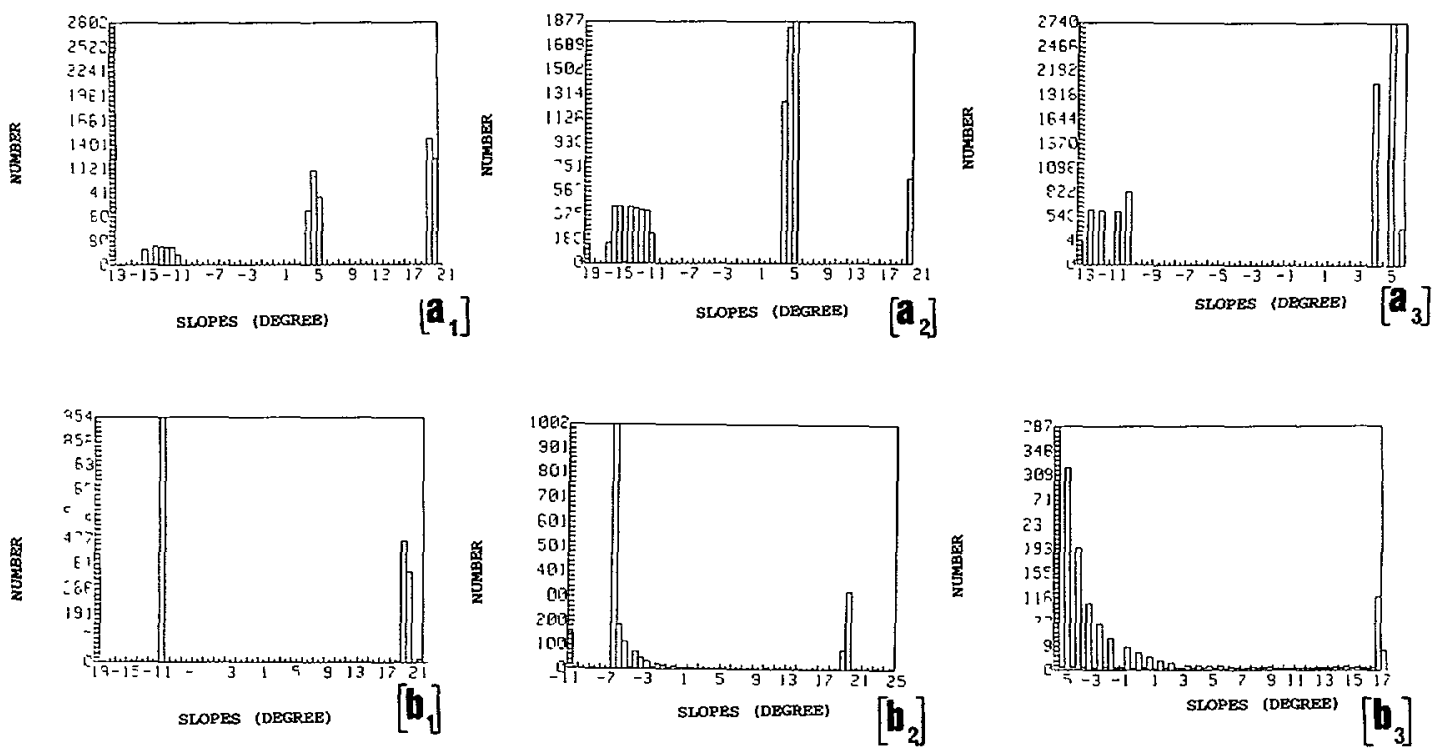

Fig. 12. - Changes in the distribution of slopes with the duration of etching. (a) $\theta=-29^{\circ} ;\left(a_{1}\right) t=4$, (a $) t=8,\left(a_{3}\right) t=12$; (b) $\theta=-79^{\circ} ;\left(b_{1}\right) t=0.1,\left(b_{2}\right) t=0.3,\left(b_{3}\right) t=1$.

i.e. a curved convex profile for the elements with positive slopes and a curved concave portion for the elements with negative slopes. We observe that the etched profiles displayed in figure $10 \mathrm{c}$ agree well with this prediction. We collate other informations from the $\beta^{*}$ vs. $\alpha$ plot of figure $13 \mathrm{c}$ : 

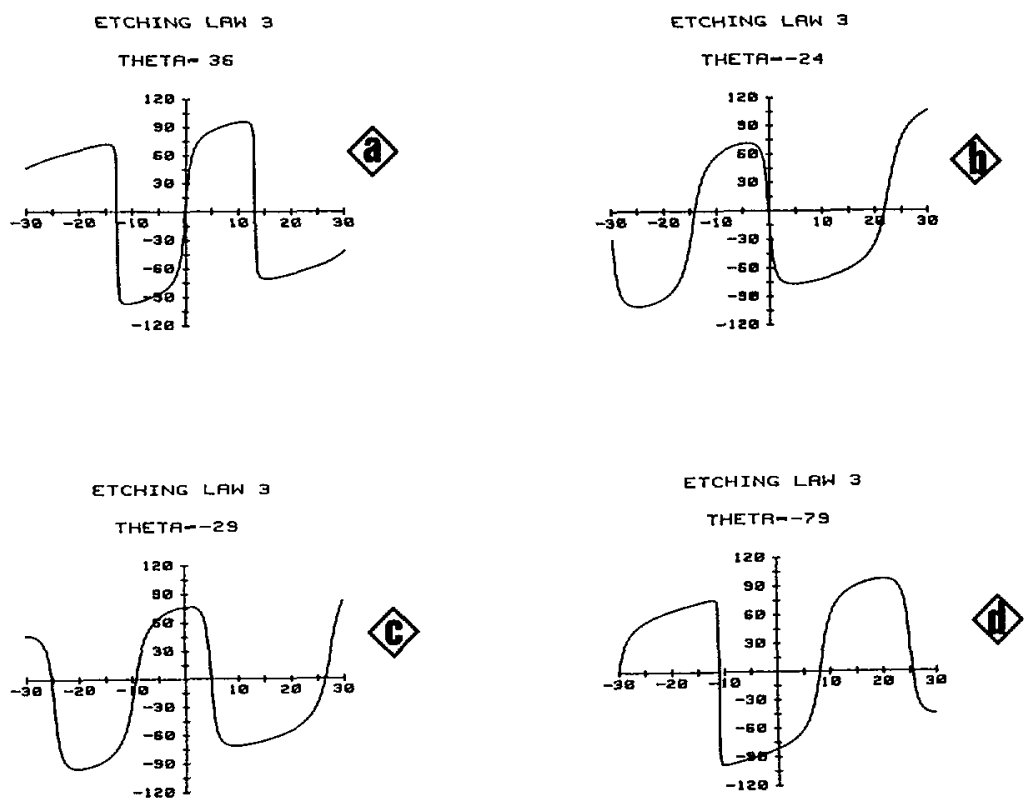

Fig. 13. - Charasteristic trajectories of the moving surface profiles elements : plots of $\beta^{*} v s$. $\alpha$ for various orientations of the reference surface. (a) $\theta=36^{\circ}$; (b) $\theta=-24^{\circ}$; (c) $\theta=-29^{\circ}$; (d) $\theta=-79^{\circ}$.

A) The curve in figure $13 \mathrm{c}$ passes through points with $\beta^{*}=0$ for $\alpha$ values close respectively to $-10^{\circ}$ and $5^{\circ}$. The diverging trajectories around $5^{\circ}$ are produced in a narrow range of $\alpha$ values whereas around $-10^{\circ}$ we are concerned with a wider range. The distributions of slopes in figure $12 \mathrm{a}$ which exhibit two distinct peaks centered on about $-10^{\circ}$ and $+5^{\circ}$ reflect very well these features.

B) Figure $13 \mathrm{c}$ contains also an information which explains why we do not observe elements with slopes in the range $\left[-8^{\circ}, 0^{\circ}\right]$ in etched profiles : this behavior can be understood in terms of trajectories moving nearly parallel to the initial surface element for $\alpha$ in the range $\left[-8^{\circ}, 0^{\circ}\right]$ so that corresponding elements do not penetrate deeply in the crystal in contrast with elements with $\alpha$ in a range close from $\left[-17^{\circ},-11^{\circ}\right]$ and $\left[2^{\circ}, 5^{\circ}\right]$ which possess nearly horizontal trajectories.

The $L v s . \alpha$ plot of figure 9d gives the etched profiles of figure $10 \mathrm{~d}$ which apparently do not follow a similar pattern. From a rapid examination of figure $9 \mathrm{~d}$ the etched profiles may be expected to exhibit an alternate concave-convex shape with a very narrow angle range over which the convexity develops. Figure $10 \mathrm{~d}$ reveals a deviating behavior with the formation in the last stages of etching of a rather « concave-concave » profile. This situation is due to the fact that for $\alpha$ in the vicinity of $-11^{\circ}$ profile elements have nearly horizontal trajectories (Fig. 13d) and etch very fastly. These elements move very rapidly within the triangular region of the crystal and with incremental etching the elements with slopes in the range $\left[-20^{\circ},-11^{\circ}\right]$ are lost. A short time after elements with slopes in the range $\left[-11^{\circ}, 0^{\circ}\right]$ contribute to the etched profile leading to a concavity. Moreover the concavity associated with positive slopes appears late because of the larger dissolution slowness and of a wider range of diverging trajectories (Fig. 13d).

To outline the role played by the relative amplitude of successive maxima and minima on the effectiventss of diverging trajectories we can compare the preceding results (Fig. 10d) 
with those derived by shifting the $L v s . \alpha$ plot of figure $9 \mathrm{~d}$ by a constant amount. It should be mentionned that adding a constant term $L_{0}$ to the angular dependent term does not modify the derivative $F^{\prime}(\gamma)=\partial F / \partial \gamma$ which characterizes the direction of motion of successive elements. A consequence is illustrated in figure $14 \mathrm{a}$ where we clearly see that the diverging trajectories concern a broader range of values for $\alpha$. The corresponding result is shown in figure 14b which undoubtely indicates slightly diverging trajectories together with an enlargement of the amount of divergence (i.e. of the $\alpha$ range, Fig. 14c). Then we observe the development of two curved portions between $\left[-20^{\circ},-10^{\circ}\right]$ and $\left[4^{\circ}, 16^{\circ}\right]$ respectively (Fig. 14c) leading to profiles with a more distinct alternate shape (Fig. 14b).
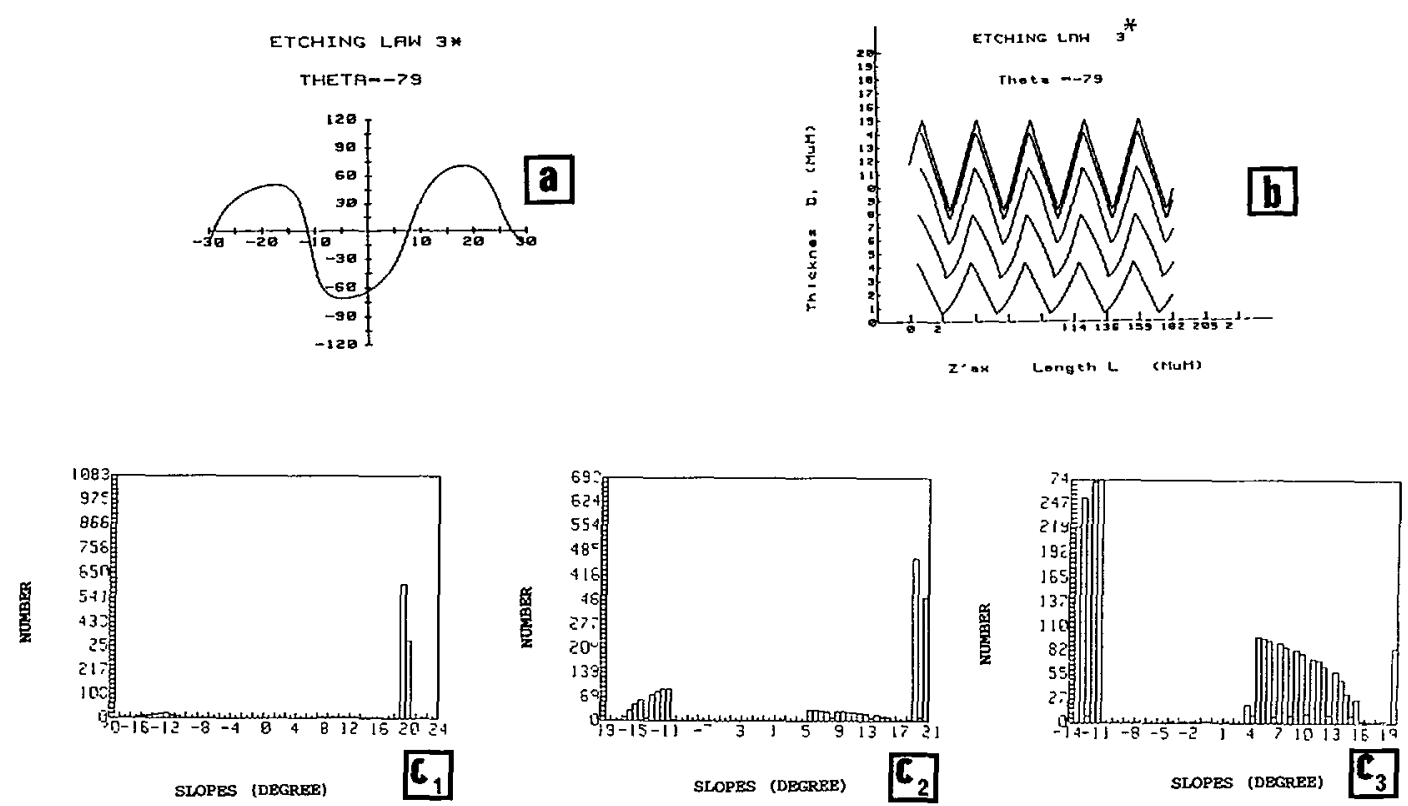

Fig. 14. - Influence of the less marked accentuation in extrema of $\mathbf{L}\left(\theta=-79^{\circ}, L_{0}=4.2\right.$ a.u. $)$ on : (a) the angle $\beta^{*}$; (b) the shape of etched profiles ; (c) the distribution of slopes : $\left(c_{1}\right) t=4,\left(c_{2}\right) t=16,\left(c_{3}\right)$ $t=48$.

Another important question is whether or not differences in the initial shape of profiles induce important changes in the etched shapes. To answer this question let us derive successive dissolution shapes starting from triangular profiles with $\alpha_{\max }$ equals respectively $10^{\circ}, 20^{\circ}$ and $30^{\circ}$. The $\beta *$ vs. $\alpha$ plot (Fig. 13a) indicates that for $\alpha$ in the range $\left(-30^{\circ}, 30^{\circ}\right.$ ) we have to take into account the highly non-meeting trajectories around $\alpha_{\mathrm{m} 1} \approx-13^{\circ}$ and $\alpha_{\mathrm{m} 2} \approx 12^{\circ}$ which for short etching time are certainly connected to slightly curved convex portions of the dissolution profiles. Effectively, figure $15 \mathrm{~b}$ reveals that etching causes the early formation of a convex intersection crudely formed by the two limiting elements associated with the two minima $\alpha_{\mathrm{m} 1}$ and $\alpha_{\mathrm{m} 2}$. In accord with this behavior the corresponding distributions of slopes (Fig. 16b) present two peaks for $\alpha$ in the vicinity of these angles. With prolonged etching (i.e. as soon as the convex intersection is only composed of the two limiting elements) we observe as expected the development of a curved concave intersection directly correlated with the maximum of $L$ occuring for $\alpha_{M} \approx 0^{\circ}$. The etching of an initial profile with $\alpha_{\max }=20^{\circ}$ leads to a similar situation (Figs. $15 \mathrm{a}$ and $16 \mathrm{a}$ ) except that in the last stage of 

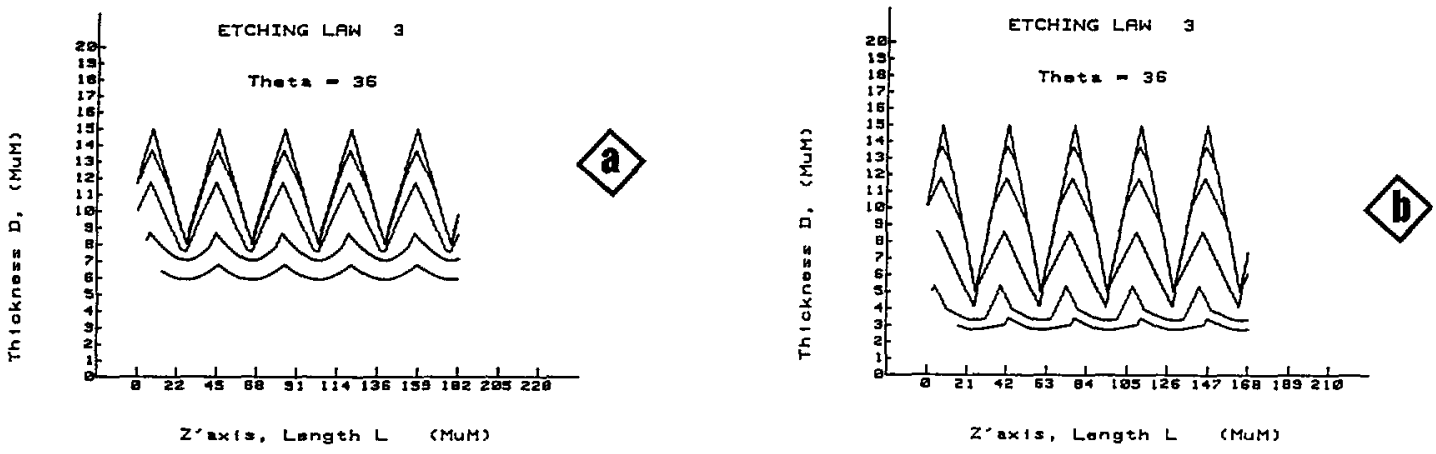

Fig. 15. - Influence of the roughness of the starting surface profile. The case where $\theta=36^{\circ}$. (a) $\alpha_{\max }=20^{\circ}$, and $t=0.2,0.5,1,2$; (b) $\alpha_{\max }=30^{\circ}$, and $t=0.2,0.5,1,1.5,2$.
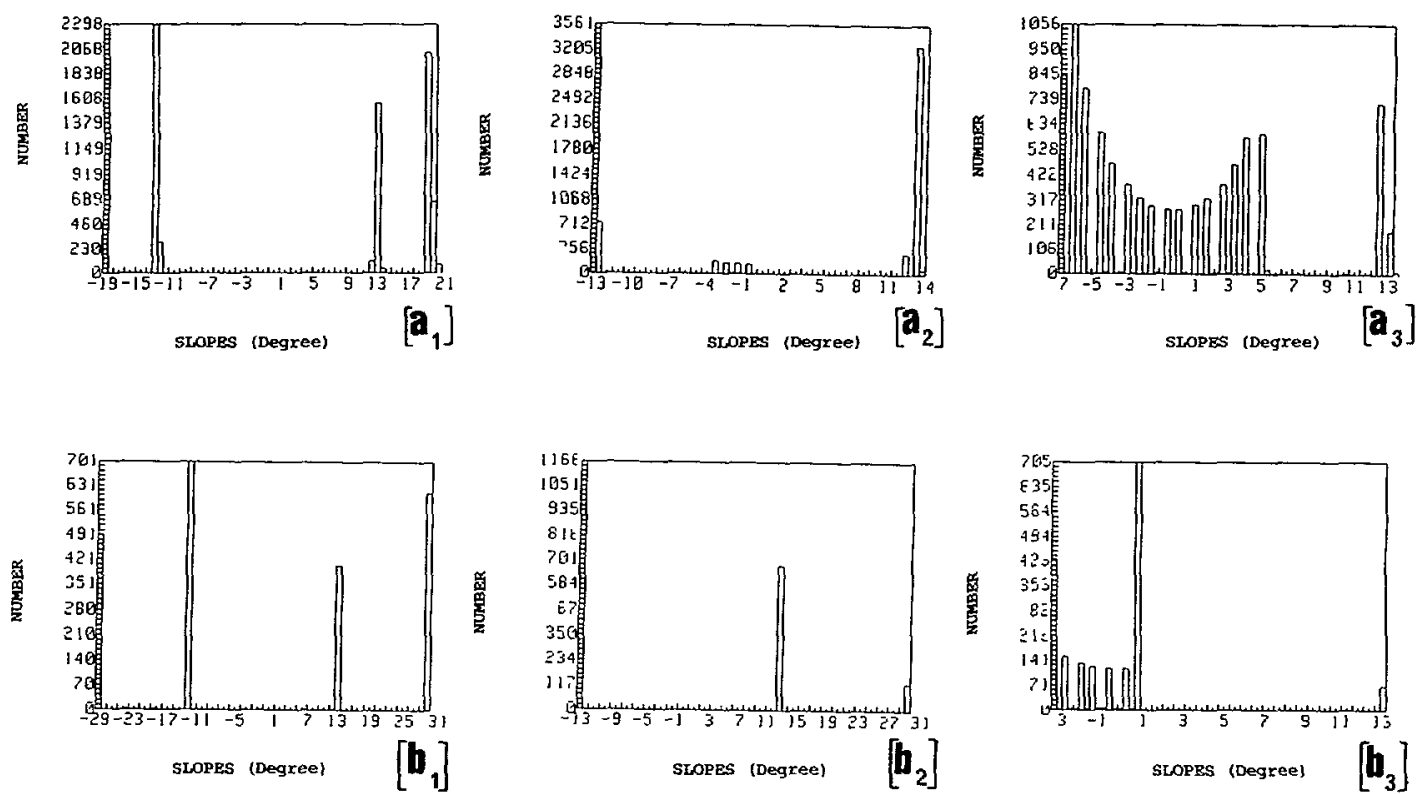

Fig. 16. - Changes in the distribution of slopes with $t$ and $\alpha_{\max }$. the case where $\theta=36^{\circ}$. (a) $\alpha_{\max }=20^{\circ},\left(\mathrm{a}_{1}\right) t=0.2,\left(\mathrm{a}_{2}\right) t=0.5,\left(\mathrm{a}_{3}\right) t=1$; (b) $\alpha_{\max }=30^{\circ},\left(\mathrm{b}_{1}\right) t=0.5,\left(\mathrm{~b}_{2}\right) t=1,\left(\mathrm{~b}_{3}\right) t=2$.

etching the curved concave portion seems to be composed of elements lying in a narrower range. However since the slopes of two successive potentially present elements are separated by $0.5^{\circ}$ in figure $16 \mathrm{~b}$ and by $1.2^{\circ}$ in figure 16a this effect may be less pronounced than that observed by comparison of these two figures.

From this study we thus infer that provided we choose a long etching time the initial surface damage does not markedly influence the final shape of etched profiles. In contrast the results in the first stage of etching depend on the starting shape of profiles.

3.2.2.2 Real surface profiles. - In the preceding we have considered that a surface profile may be approached by a triangular profile. However a real surface profile possesses 
geometrical imperfections which generally take the form of peaks and valleys of varying height and width [52]. This is in particular the case for surfaces produced by lapping. Then it is of interest to apply the numerical simulation to a digitalized surface profile as worked by a microprocessor based profilometer. Two real surface profiles resulting from two different lapping processes are given in figure 17. The corresponding distribution of slopes are crudely symmetrical and may be recognized as gaussian distributions.

The changes in shapes and in slope histograms with increasing etching time are depicted in figures 18 to 22 for two different orientations of the reference surface, various etching laws and/or different initial surface damages. Then for convenience the discussion is divided in two parts.

3.2.2.2.1 Reference surface with $\theta_{0}=36^{\circ}$. - Turning our attention to figure $17 \mathrm{~b}$ we observe that the slope angles are for a large majority in the range $\left(-20^{\circ}, 20^{\circ}\right)$. One can then expect that for long etching times we work essentially with angles in the vicinity of the maximum $\alpha_{M}$ (Fig. 9a) which is known to give rise to a final concave background. It is this behavior which emerges from figure 18 where a relatively flat quasi concave section develops as soon as the few elements with slope angles around $-13^{\circ}$ and $12^{\circ}$ which etch faster are removed. All the features illustrated in figure 18 agree with the conclusions drawn in section 3.2.2.1. In particular for relatively short etching times we obtain a distribution of slopes composed of three peaks. The «platy kurtic » nature of the secondary peaks can be understood in terms of a small number of elements with slope in the ranges $\left(-20^{\circ},-10^{\circ}\right)$ and $\left(10^{\circ}, 20^{\circ}\right)$.

Now changing the etching law (Fig. 23) in order to obtain diverging trajectories on wider ranges of slope angles gives etched profiles which in the first stage of etching present some convex portions (see for example regions $H_{1}$ and $H_{2}$ in Figs. $19 \mathrm{a}_{1}$ and $19 \mathrm{a}_{2}$ ) for
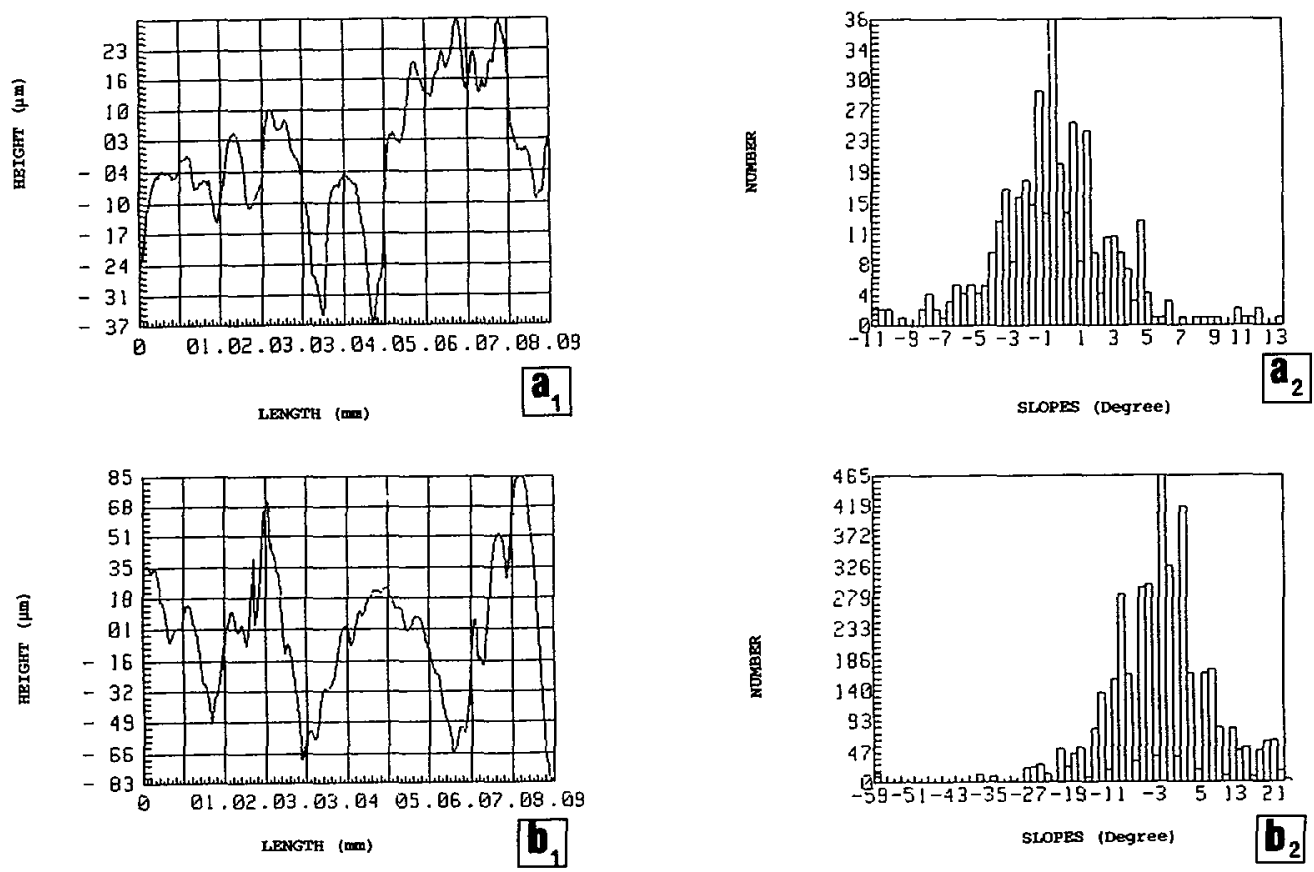

Fig. 17. - Real profilometry traces on differently lapped surfaces : profile shapes and slopes distributions. (a) average diameter of abrasive grains : $D_{g}=5 \mu \mathrm{m}$; (b) average diameter of abrasive grains: $D_{\mathrm{g}}=10 \mu \mathrm{m}$. 


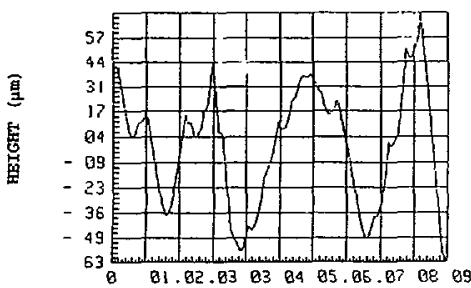

LENGTH (mm)

$\left[a_{1}\right]$

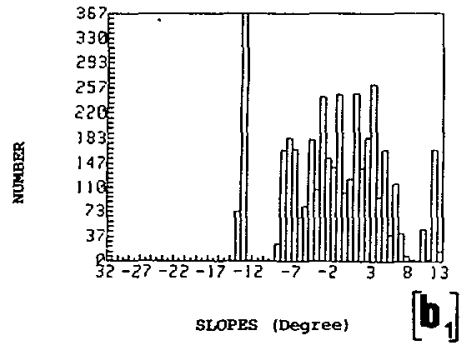

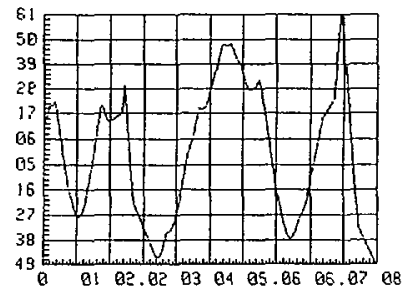

$\left[a_{2}\right]$

LENGTH (man)

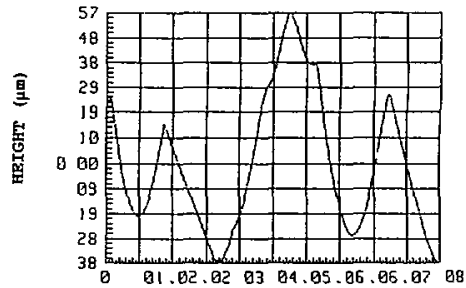

$\left[\mathbf{a}_{3}\right]$

LENGTH (ma)
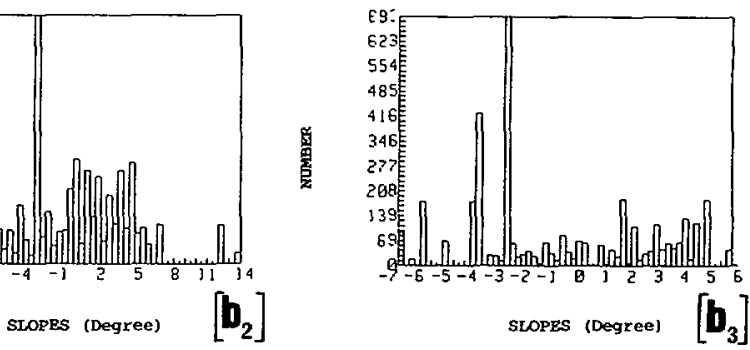

SLOPES (Degree)

$\left[b_{3}\right]$

Fig. 18. - Etching of a lapped surface $\left(D_{\mathrm{g}}=10 \mu \mathrm{m}\right)$ of orientation $\theta=36^{\circ}$. (a) Theoretical shapes of etched profiles : $\left(a_{1}\right) t=0.1 ;\left(a_{2}\right) t=0.2 ;\left(a_{3}\right) t=0.5$. (b) Theoretical distributions of slopes : $\left(b_{1}\right)$ $t=0.1 ;\left(\mathrm{b}_{2}\right) t=0.2 ;\left(\mathrm{b}_{3}\right) t=0.5$.
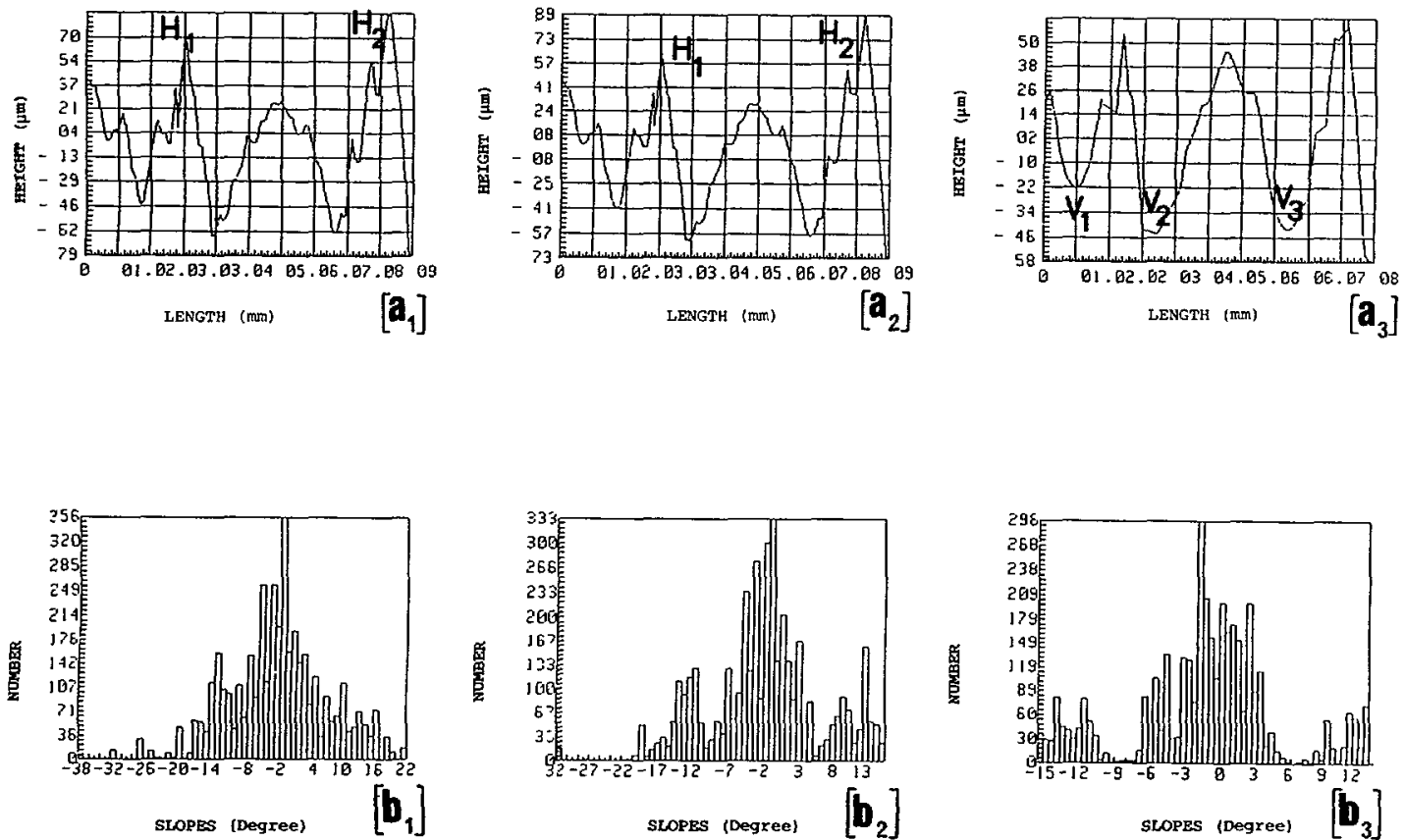
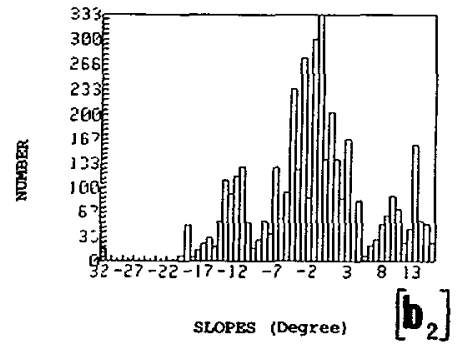

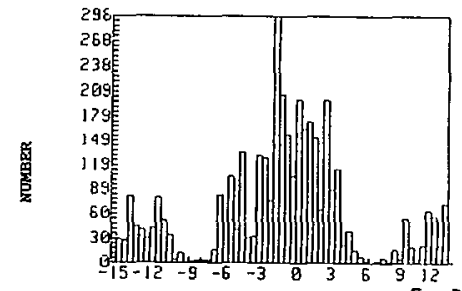

SLOPES (Degree)

Fig. 19. - Etching of a lapped surface $\left(D_{g}=10 \mu \mathrm{m}\right)$ of orientation $\theta=36^{\circ}:$ the case where the extrema of $L$ are less accentuated. (a) Theoretical shapes of etched profiles : $\left(\mathrm{a}_{1}\right) t=4 ;\left(\mathrm{a}_{2}\right) t=8,\left(\mathrm{a}_{3}\right)$ $t=24$. (b) Theoretical distributions of slopes: $\left(\mathrm{b}_{1}\right) t=4 ;\left(\mathrm{b}_{2}\right) t=8,\left(\mathrm{~b}_{3}\right) t=24$. 

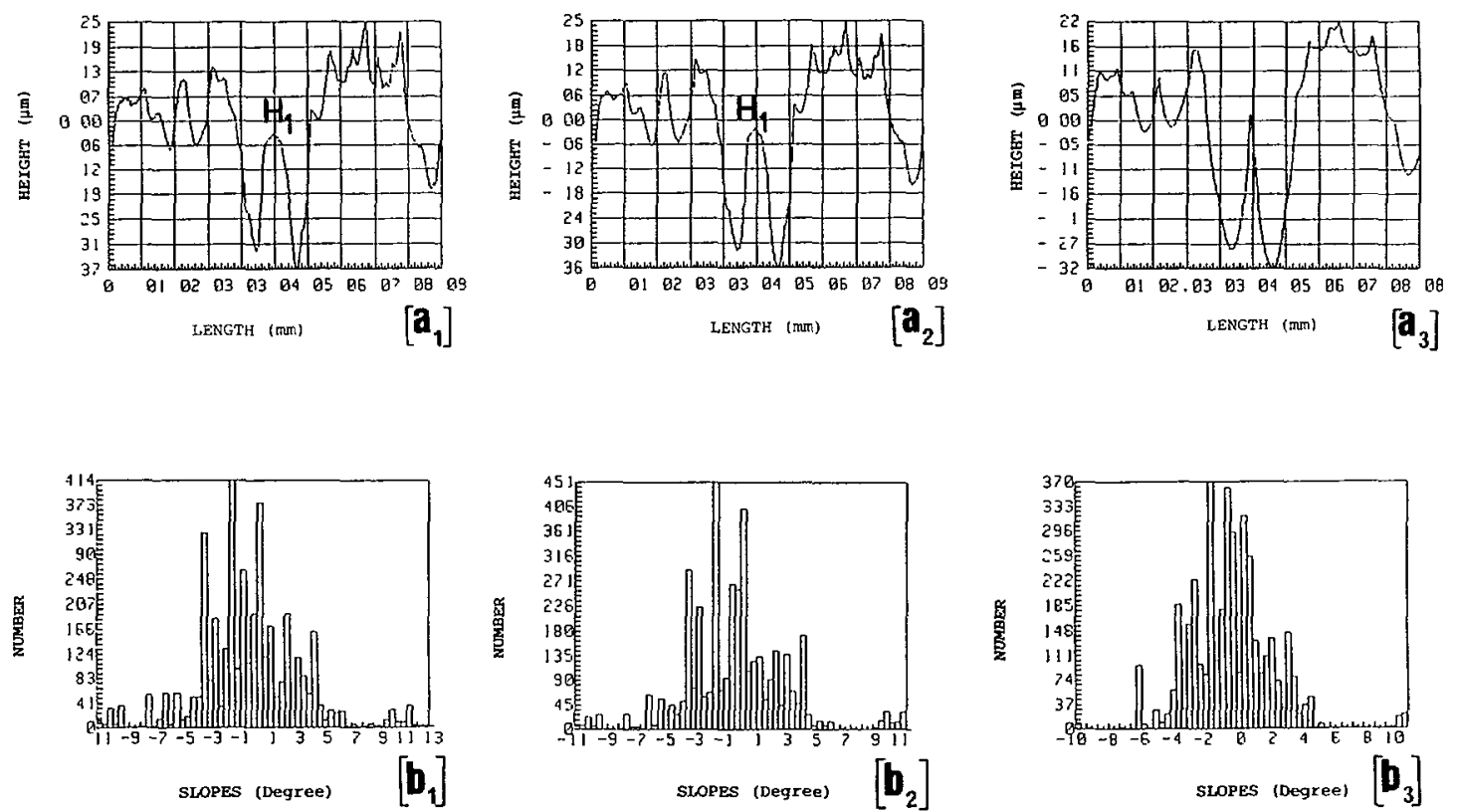

Fig. 20. - Etching of a lapped surface $\left(D_{\mathrm{g}}=5 \mu \mathrm{m}\right)$ of orientation $\theta=36^{\circ}$ : the case where the extrema of $L$ are less accentuated $\left(L_{0}=4.2\right.$ a.u. $)$. (a) Theoretical shapes of etched profiles $\left(a_{1}\right) t=4,\left(a_{2}\right) t=8$, $\left(a_{3}\right) t=24$; (b) Theoretical distributions of slopes $\left(b_{1}\right) t=4,\left(b_{2}\right) t=8,\left(b_{3}\right) t=24$.

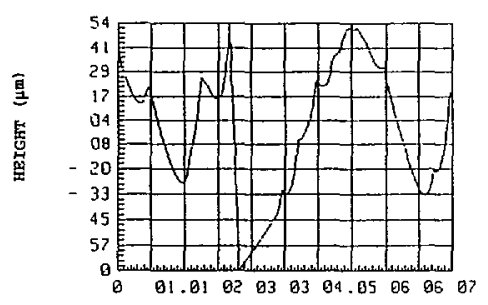

$\left[a_{1}\right]$

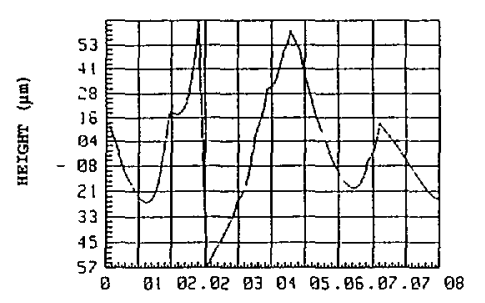

$\left[\mathbf{a}_{2}\right]$

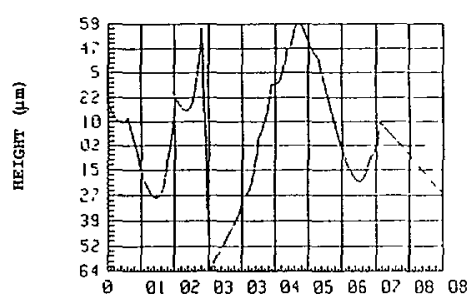

LENGTH (mM)

$\left[\mathbf{a}_{3}\right]$
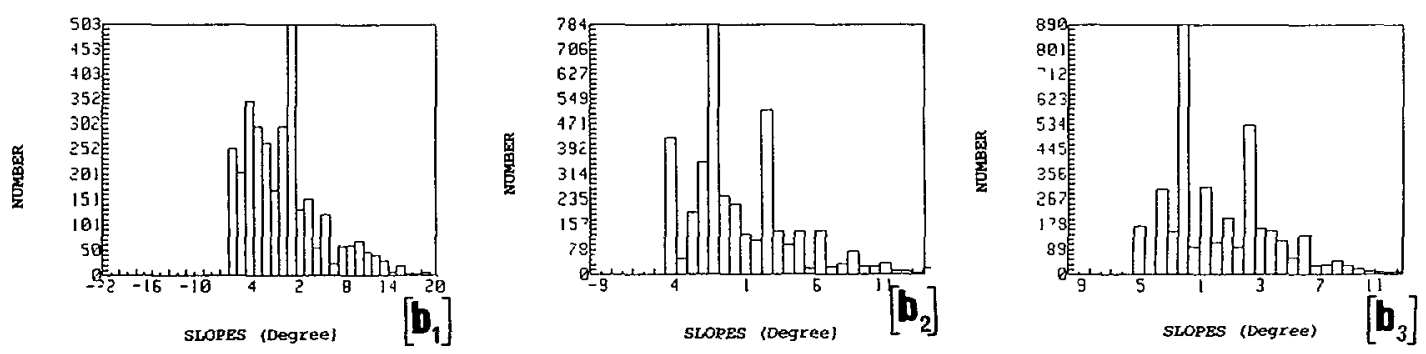

Fig. 21. - Etching of a lapped surface $\left(D_{\mathrm{g}}=10 \mu \mathrm{m}\right)$ of orientation $\theta=-79^{\circ}$. (a) Theoretical shapes of etched profiles $\left(\mathrm{a}_{1}\right) t=0.3,\left(\mathrm{a}_{2}\right) t=0.6,\left(\mathrm{a}_{3}\right) t=1$; (b) Theoretical distributions of slopes $\left(\mathrm{b}_{1}\right) t=0.3$, $\left(\mathrm{b}_{2}\right) t=0.6,\left(\mathrm{~b}_{3}\right) t=1$. 

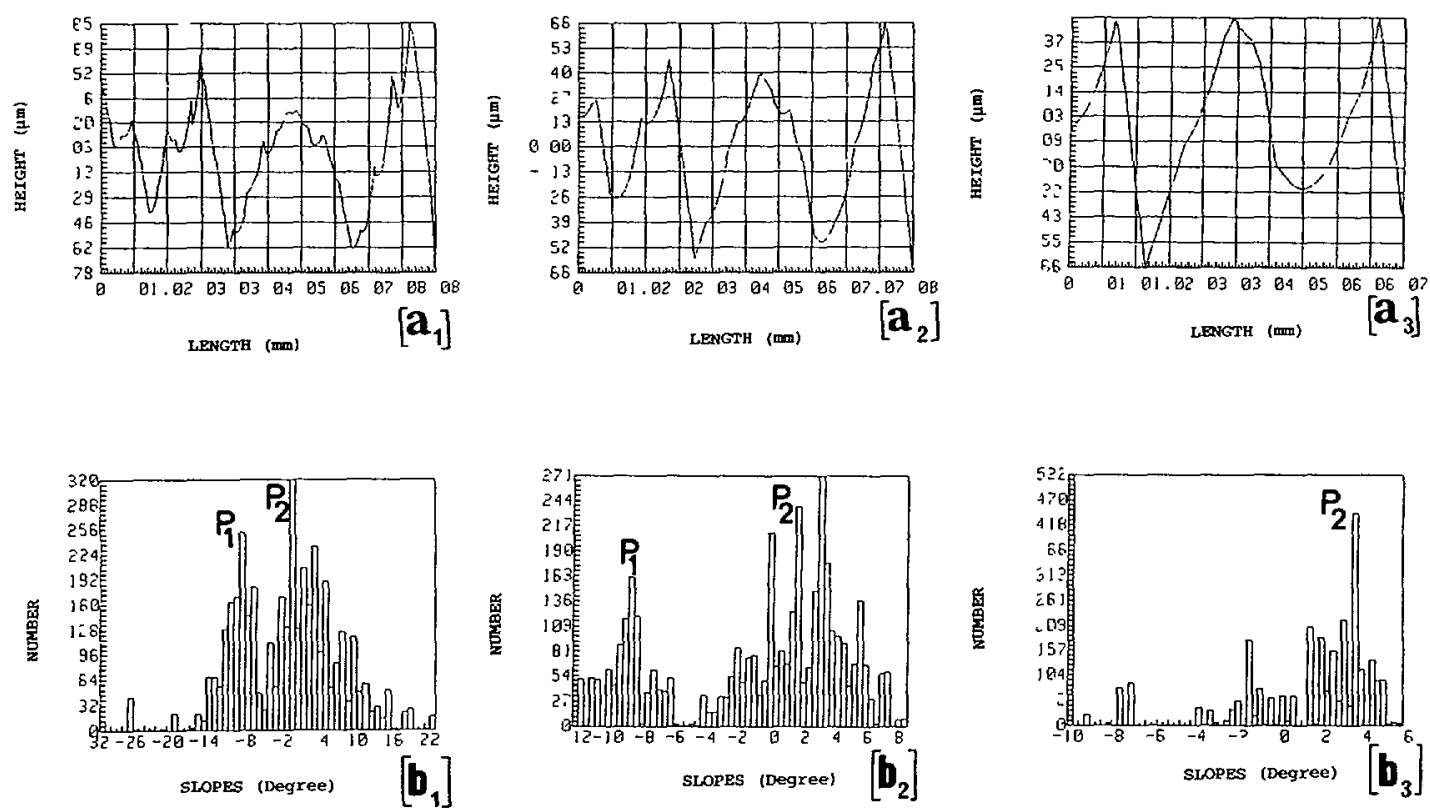

Fig. 22. - Etching of a lapped surface $\left(D_{\mathrm{g}}=10 \mu \mathrm{m}\right)$ of orientation $\theta=-79^{\circ}:$ the case where the extrema of $L$ are less accentuated $\left(L_{0}=4.2 \mathrm{a}\right.$.u.). (a) Theoretical shapes of etched profiles $\left(\mathrm{a}_{1}\right) t=4$, $\left(a_{2}\right) t=16,\left(a_{3}\right) t=48$; (b) Theoretical distributions of slopes $\left(b_{1}\right) t=4,\left(b_{2}\right) t=16,\left(b_{3}\right) t=48$.

$\alpha$ around $\alpha_{\mathrm{m} 1}$ or $\alpha_{\mathrm{m} 2}$. In the corresponding distribution of slopes (Figs. $19 \mathrm{~b}_{1}$ and $19 \mathrm{~b}_{2}$ ) two secondary peaks are effectively initiated by these two minima in $L$ which develop with prolonged etching. As a consequence it becomes more difficult to distinguish exact final shape of dissolution profile : i.e. to decide between a totally concave section and a section witr alternate shape (see portion $V_{1}$ or $V_{3}$ in Fig. $19 \mathrm{a}_{3}$ ).

Turning to the surface profiles displayed in figure $17 \mathrm{a}$ it is clear that in the first stages of etching the convex curved portions of profiles (see elements around $H_{1}$ in Figs. 20a $a_{1}$ and $20 \mathrm{a}_{2}$ ) are associated with the few initial slopes in the vicinity of $\alpha_{\mathrm{m} 1}$ and $\alpha_{\mathrm{m} 2}$. These convex portions disappear with further etching and the final etched profile exhibits a concave shape as expected.

3.2.2.2.2 Reference surface with $\theta_{0}=-79^{\circ}$. - Let us be concerned with the etching law shown in figure $9 \mathrm{~d}$ and with an initially lapped surface (Fig. 17b). In the first stage of etching we expect the successive etched shapes of the profiles to be primarely determined by the presence of the pronounced minimum $\alpha_{\mathrm{m} 1}$ in $L$ which may induce a curved convexity for elements with negative slopes lying in the close vicinity of $-11^{\circ}$. In fact (Fig. 21) at the first sight the etched profiles appear to be like a concave background structure. Figures $21 b_{1}, b_{2}$, $b_{3}$ are a little more instructive than figures $21 a_{1}, a_{2}, a_{3}$. the assymetry of the distribution as well as the absence of elements with slopes around $0^{\circ}$ can inform us on the «false » curved concave aspect of etched profiles.

If we modify the etching law (Fig. 14a) in such a manner that the extrema of $L$ are less accentuated and that the reference surface dissolves less rapidly the formation and the persistence of elements with slopes around $-11^{\circ}$ is now clearly favoured (Figs. $22 b_{2}, b_{3}$ ). As a result we obtain dissolution profiles with alternate concave-convex shapes (Figs. 22 $\mathrm{a}_{1}$, $\left.a_{2}, a_{3}\right)$. 


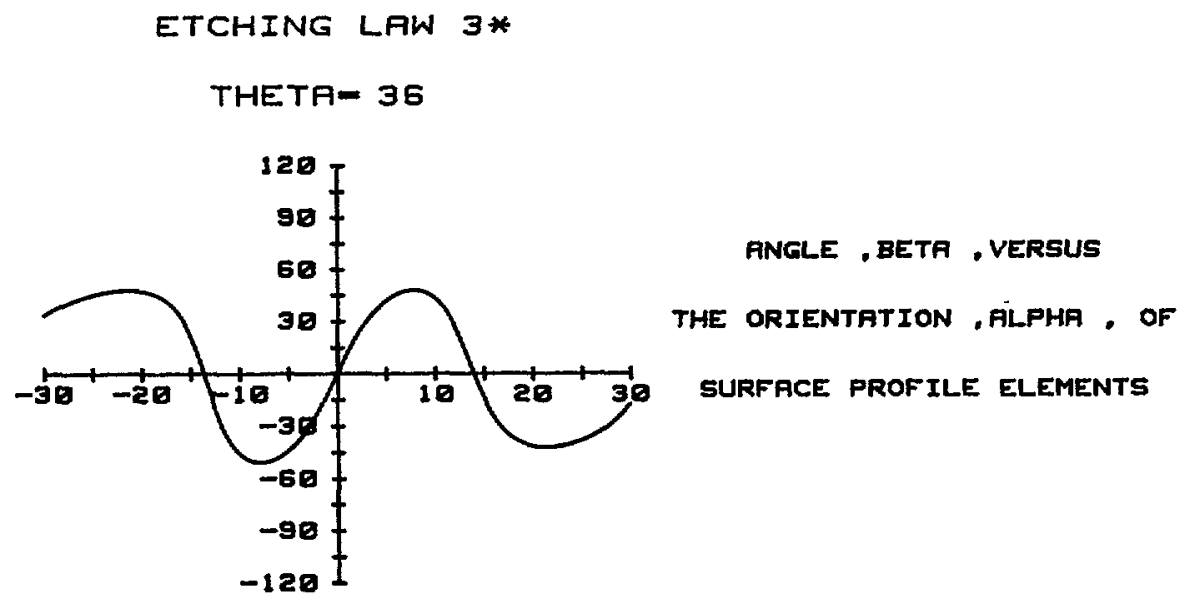

Fig. 23. - The $\beta^{*} v s . \alpha$ plot in the case of a modified etching law with less accentuated extrema.

Turning to figures $22 b_{1}, b_{2}, b_{3}$ we observe that the distributions of slopes exhibit two distinct peaks $\mathrm{p}_{1}$ and $\mathrm{p}_{2}$ around $\alpha \approx-10^{\circ}$ and $\alpha \approx 6^{\circ}$. Then the position of the peak $\mathrm{p}_{1}$ allows us to identify quite accurately the orientation $\alpha_{\mathrm{m} 1}$ corresponding to a minimum in $L$. But the precise determination of the angle $\alpha_{M 1}$ associated with the maximum remains difficult because of a broader range of diverging trajectories : the peak $p_{2}$ which is associated to the concave curved portions of profiles concerns effectively angles in the range $\left[-4^{\circ}, 10^{\circ}\right]$. We also observe that with prolonged etching the number of elements with slopes around $0^{\circ}$ decreases in accord with the formation of a profile of rather concave-convex shape presenting sharp discontinuities at convex intersection.

\section{Discussion and conclusion.}

The construction of successive dissolution shapes of starting cylindrical hollows or crystals from differently oriented polar diagrams of the dissolution slowness can be accomplished by numerical simulation. It permits to establish some features essential for the converse use of the theory i.e. for the approximate determination of the shape for the dissolution slowness surface. These features can be summarized as follows:

1) There is a crude resemblance in shape between the out-of-roundness profiles and the corresponding polar diagrams.

2) From the out-of-roundness profiles of an initially cylindrical hollow we can establish for what orientations the slowness vector $\mathbf{L}$ rotating in the cross-sectional plane passes through successive maxima. Conversely from out-of-roundness profiles referred to a cylindrical crystal we deduce the exact orientations connected to minima.

3) For these extrema, called for convenience principal extrema, which produce limiting facets we can evaluate with a good approximation the magnitude, $L(\varphi, \theta)$, of the dissolution slowness vector.

4) In general we cannot recognize precisely the orientation $(\varphi, \theta)$ of surface elements associated with the sharp discontinuities in the out-of-roundness profiles because crystal edges are produced by intersecting trajectories which change with incremental etchings.

5) Measurements of the «apparent » dissolution slowness related to very sharp discontinuities do not yield the true value of $L(\varphi, \theta)$ connected to extrema whose nature is converse to that of principal extrema. 
6) Deviation from the behavior described in 4 (i.e. the independence of the orientation of crystal edges with repeated etching) can be understood in terms of crudely symmetrical portions of the polar diagram.

When we turn to the problem of determining the slowness surface of a crystal we have several remarks to point. Firstly let us recall that in the one hand there are selection rules between the $N_{\mathrm{c}}$ unknown coefficients appearing in the generalized equation of the dissolution slowness and that on the second hand we have generally serious difficulties to measure precisely the etch rate of differently-oriented crystals. For these reasons a computation of the slowness surface by solving a system of $N_{\mathrm{c}}$ equations makes frequently the resulting shape uncertain. An alternative approach consists of choosing values for the dissolution constants rather than computing values for the unknown coefficients. The symmetry relations between the unknown coefficients are followed by this procedure which in fact includes successive iterations before leading to a satisfactory result and implies a further procedure to verify if the derived shape is fully appropriate. We first decide to stop the procedure of generation of the slowness surface when, according to the preceding features 1,2 and 3, various cross-sectional shapes of the slowness surface are in crude agreement with corresponding experimental outof-roundness profiles. Such a comparison allows us to verify the orientations $\left(\varphi_{\mathrm{e}}, \theta_{\mathrm{e}}\right)$ for which the dissolution slowness passes through extrema. A complete determination of all the angles $\left(\varphi_{e}, \theta_{\mathrm{e}}\right)$ requires evidently that experiments are performed simultaneously on cylindrical hollows and crystals. It is in general sufficient to perform experiments on less than ten differently oriented crystals provided they are large deviations between the angles of cut. But with this procedure we are not sure that a minor perturbation in the slowness surface can be detected. Effectively the least accentuated extrema give rise to facets of small extent which cannot be easily recognized on the out-of-roundness profiles. In practice to track sligth irregularities in the slowness surface we need to obtain complementary informations from two sets of experiments :

I) from examination of various cross-sectional profiles related to localized etchings at a mask ;

II) from a systematic study of the changes, with orientation, in the final etched shapes of surface profiles.

The first set of experiments allows us to recognize without ambiguity the influence of a minor maximum in $L$. From SEM observations it is possible to evaluate with a satisfactory accuracy the corresponding orientation $\left(\varphi_{\mathrm{M}}, \theta_{\mathrm{M}}\right)$. But such experiments are limited to the maxima in $L$ and requires processes of high technology (photolithography process, S.E.M. examination.. ). Moreover we have to select judiciously the orientations of the cross-sections to reduce the number of experiments.

Observation of the etched shape of surface profiles can furnish additional possibilities such as :

a) An easy verification of the orientation of maxima and minima in $L$.

b) Evidence of the presence of a minor extremum in the vicinity of the orientation $\left(\varphi_{0}, \theta_{0}\right)$ of a reference surface, independently of the nature of the extremum.

We can extend these possibilities by following simultaneously the evolution of the distribution of slopes with the angles of cut $\left(\varphi_{0}, \theta_{0}\right)$ and the duration of etching :

c) The symmetry of peaky slope distributions together with a peak centered about $\alpha=0^{\circ}$ indicates that an extremum of $L$ occurs for an orientation $\left(\varphi_{0}, \theta_{0}\right)$ which coincides with that of the reference surface and that the polar diagram is symmetrical in the $\alpha$-range investigated here (i.e. the range of starting slopes). Any marked asymmetry in the polar diagram induces an asymmetric spread in the distribution of slopes. 
d) The symmetry of the distribution of slopes together with a lack of slopes around $\alpha=0^{\circ}$ may be attributed to the presence, in the $\alpha$-range of two extrema of converse nature which lie «symmetrically » with respect to $\alpha=0^{\circ}$. These extrema induce the formation of two supplementary peaks whose positions can move with repeated etchings.

e) Negatively and positively skewed slope distributions together with a spread which extends asymmetrically to large angles are interpreted in terms of a shift of one of the two extrema toward these angles.

f) The development of two distinct separate peaks respectively located at $-\alpha_{1}$ and $\alpha_{2}$ together with the persistence with prolonged etching of a «leptokurtic » peak at $\alpha \approx 0^{\circ}$ results from a polar diagram more complex than that causing the features described in d) [2]. Three extrema are also involved in the present case.

g) Because of highly diverging trajectories very accentuated extrema give rapidly rise to relatively flat etched profiles. Conversely relatively «smooth » extrema cause the formation of etched profiles composed of elements with higher slopes. Then we can, with use, distinguish between sharp and smooth extrema. Provided there is no difference between the initial surface damages of various plates a crude estimation of the relative amplitude of successive extrema remains possible.

Taking into account all the features presented in this section we can propose a complete procedure to generate the dissolution slowness surface of a crystal. This procedure consists successively of :

1) Generating by iterations the slowness surface until various theoretical polar diagrams of $L$ resemble to corresponding experimental out-of-roundness profiles.

2) Verifying the adequation of the derived slowness surface. This verification consists essentially of a comparison of experimental etched shapes with predicted shapes. After selecting some interesting orientations to perform experiments three possibilities are offered to the investigator :

(i) etching of cylindrical hollows and crystals (determination of orientations $\varphi_{\mathrm{e}}, \theta_{\mathrm{e}}$ )

(ii) localized etching at a mask (finding of minor perturbations)

(iii) etching of rough surfaces (verification of the positions of extrema and study of the accentuation of extrema).

In case (iii) it is necessary to produce starting lapped surfaces with identical geometrical surface features (roughness parameters and distributions of slopes).

It is also essential to estimate the degree of accuracy in the amplitude of some extrema by measurements of the etch rate of thin plates with orientations $\left(\varphi_{\mathrm{e}}, \theta_{\mathrm{e}}\right)$. In this case the workers have to regard the following points :

A) In the first stages of etching the initial surface damage of rough surfaces induces an increase in the etch rate. Carefully polished surfaces and a prolonged etching are thus needed.

B) The measurements must be perform on plates for which the two surfaces have by symmetry considerations the same etch rate.

The most important conclusion is that the problem of deriving the true shape for the dissolution slowness surface is not intractable but it needs attention when analysing experimental etch shapes or etch rate data. Fortunately the investigators without ignoring the complexity of the problem can undertake various complementary experiments in an endeavour to adjust the theoretical shape of the slowness surface. An attempt will be made in a future paper to illustrate this procedure and to derive the slowness surface of quartz crystal from experimental data. 


\section{References}

[1] Sangwal K., « Etching of Crystals » (North-Holland, Amsterdam, 1987).

[2] IRVING B. A., in « The Electrochemistry of Semiconductors », edited by P. J. Holmes (Academic Press, London, 1962) pp. 256-289.

[3] Heimann R. B., in « Silicon Chemical Etching », edited by J. Grabmaier (Springer, Berlin, 1982).

[4] Wegner M. W. and Christie J. M., Phys. Chem. Minerals 9 (1983) 67.

[5] Tellier C. R., Surf. Technol. 21 (1984) 83.

[6] Gatos H. C. and Lavine M. C., J. Electrochem. Soc. 107 (1960) 433.

[7] Castagliola M., Tellier C. R, and Vaterkowski J. L., J. Mater. Sci. 21 (1986) 3551.

[8] FAust J. W., in « The Surface Chemistry of Metals and Semiconductors » (edited by H. C. Gatos, Wiley, New York, 1960) p. 151.

[9] Holmes P. J., in « The Electrochemistry of Semiconductors ", edited by P. J. Holmes (Academic Press, London, 1962) pp. 329-377.

[10] Tellier C. R. and Jouffroy F., J. Mater. Sci. 18 (1983) 3621.

[11] Tellier C. R., Jouffroy F. and Buron C., Mater. Chem. Phys. 14 (1986) 25.

[12] Tellier C. R. and VAterkowski J. L., Surf. Technol. 26 (1985) 275.

[13] Tellier C. R., Vialle N. and Vaterkowski J. L., Proceedings 40th Annual Symposium on Frequency Control, Philadelphia, Pa, 1986 (IEEE, New York, 1986) p. 76.

[14] Tellier C. R., Vialle N. and Vaterkowski J. L., First European Time and Frequency Forum, Besançon, France, 1987 (Imprimerie du Conseil Général du Doubs, Besançon, 1987) p. 159.

[15] Vig J., WASShausen H., COOK C., KatZ M. and HafNER E., Proceedings 27th Annual Symposium on Frequency Control, Forth Monmouth, N.J., 1973 (Electronic Industries Association, Washington, DC, 1973) p. 98.

[16] VIG J. R., LEBUS J. W. and FILLER R., Report ECOM-4548, 1977 (US Army Electronics Command, Fort Monmouth, NJ).

[17] Vig J. R., Cook C. F., Schwidtal K., Lebus J. W. and Hafner E., Proceedings 28th Annual Symposium on Frequency Control, Fort Monmouth, NJ, 1974 (Electronic Industries Association, Washington, DC, 1974) p. 96.

[18] VIG J. R., BRANDMAYR R. J. and FILler R. L., Proceedings 33rd Annual Symposium on Frequency Control, Fort Monmouth, NJ, 1979 (Electronic Industries Association, Washington, DC, 1979) p. 351.

[19] BrandmayR R. J. and Vig J. R., Proceedings 39th Annual Symposium on Frequency Control, Philadelphia, Pa, 1985 (IEEE, New York, 1985) p. 276.

[20] Tellier C. R. and Leblois T. G., in Proceedings of the Third European Time and Frequency Forum, Besançon, France, 1989 (Imprimerie du Conseil Général du Doubs, 1989) pp. 246255.

[21] JACCODINe R. J., J. Appl. Phys. 33 (1962) 2643.

[22] Frank F. C. and Ives M. B., Ibid. 31 (1960) 1996.

[23] Petersen K. E., Proc. IEEE 70 (1982) 420.

[24] DelapierRe G., Sensors and Actuators 17 (1989) 123.

[25] Danel J. S., Michel F. and DelapierRe G., Sensors and Actuators A 21-A 23 (1990) 971.

[26] Choffat H., 58 ${ }^{\mathrm{c}}$ Congrès de la Société Suisse de Chronométrie, Bienne, Switzerland, 1983, Paper No. 8.

[27] Ueda T., Kohsaka F. and OGITA E., Proceedings 10th Conference IMEKO on Measurement of Force and Mass (Kobe, Japan, September 1981) pp. 17-22.

[28] ZINGG W., CNRS Seminar on Frequency Standards, Besançon, 6-7 mars 1985 (Editions du CNRS, Paris, 1985) pp. N-1-15.

[29] SHAw D. W., J. Electrochem. Soc. 128 (1981) 874.

[30] SHAW D. W., J. Crystal Growth 47 (1979) 509.

[31] Weirauch D. F., J. Appl. Phys. 46 (1975) 1478.

[32] Stoller A. I., RCA Rev. 31 (1970) 271. 
[33] LeE D. B., J. Appl. Phys. 40 (1969) 4569.

[34] BEAN K. E., IEEE Trans. Electron. Dev. ED-25 (1978) 1185.

[35] CSEPREGi L., Microelectr. Eng. 3 (1985) 221.

[36] Seidel H. and Csepregi L., Sensors and Actuators 4 (1983) 455.

[37] Seidel H., Csepregi L., Heuberger A. and Baumgartel H., J. Electrochem. Soc. 137 (1990) 3612.

[38] Mayer G. K., Offereins H. L., Sandmaier H. and KuHl K., Ibid. 137 (1990) 3947.

[39] OfFereins H. L., KUHL K. and SANDMAIER H., Sensors and Actuators A 25 (1991) 9-13.

[40] XIAN-PING WU and Wen Ko H., Ibid. 18 (1989) 207.

[41] ABU-ZeId M. M., J. Electrochem. Soc. 131 (1984) 2138.

[42] Lighthill M. and Whitman G., Proc. Roy. Soc. A 229 (1955) 281.

[43] Frank F. C. in Doremus R. H., Robert B. W. and TURnbull D. (Eds.), Growth and Perfection of Crystals (John Wiley, New York, 1958) pp. 411-419.

[44] Teliter C. R., Vialle N. and Vaterkowski J. L., Surface Coatings Technol. 34 (1988) 417.

[45] Tellier C. R. and Vaterkowski J. L., J. Mater. Sci. 24 (1989) 1077.

[46] Tellier C. R., J. Crystal Growth 96 (1989) 877.

[47] Brahim-Bounab A., Amaudrut J. Y. and Tellier C. R., J. Mater. Sci. (to be published, 1991).

[48] Tellier C. R., Amaudrut J. Y. and Brahim-Bounab A., J. Mater. Sci. (to be published, 1991).

[49] Standard on Piezoelectricity (IEEE, New York, 1978) pp. 15-27.

[50] Tellier C. R., J. Mater. Sci. 17 (1982) 1348.

[51] Batterman B. W., J. Appl. Phys. 28 (1957) 1236.

[52] Thomas T. R. (Ed.), Rough Surfaces (Longman, London, 198) Chap. 4. 